\title{
Quantifying the effects of mastitis on the reproductive performance of dairy cows: A meta-analysis
}

\author{
K. A. Dolecheck, A. García-Guerra, and L. E. Moraes* \\ Department of Animal Sciences, The Ohio State University, Columbus 43210
}

\begin{abstract}
The objective of this study was to conduct a metaanalysis to quantitatively characterize the effects of mastitis on the reproductive performance of dairy cows as well as to identify factors that interact with this relationship. A total of 29 publications were identified that contributed $24,41,27,38$, and 13 trial results to the meta-analysis of how mastitis is related to time to first service (TFS), days open (DO), services per conception (SPC), pregnancies per insemination at first service (FSP/AI) and pregnancy loss (PL), respectively. The meta-analyses were conducted using multilevel linear mixed-effects models. Overall, high levels of heterogeneity were present and meta-regression models only explained a small amount of heterogeneity. Results suggest that cows with mastitis pre-first insemination experience, on average, an additional $13.29 \mathrm{~d}$ to first service [95\% confidence interval (CI): 6.64, 19.95] when compared with cows with no mastitis in the same time period. Moreover, in relation to cows with no mastitis in the same time period, 22.34 additional DO (95\% CI: $12.89,31.79)$ were estimated, on average, for cows with clinical mastitis at pre-insemination leading to conception. Additionally, 32.41 added DO (95\% CI: $20.58,44.25)$ were estimated, on average, for cows with clinical mastitis at pre- or post-insemination leading to conception compared with cows with no mastitis in the same time period. Finally, 20.03 additional DO (95\% CI: 3.11, 36.95) were estimated, on average, for cows with subclinical mastitis pre- or post-insemination leading to conception compared with cows with no mastitis in the same time period. Effect size estimates from the meta-regression models for SPC, in relation to cows with no mastitis in the same time period, suggest that, on average, SPC increases by 0.46 inseminations (95\% CI: $0.30,0.62)$ for a cow experiencing mastitis
\end{abstract}

Received May 29, 2018.

Accepted April 25, 2019.

*Corresponding author: ferrazdiasdemoraes.1@osu.edu pre-insemination leading to conception. When mastitis occurs either pre- or post-insemination leading to conception, SPC is expected to increase, on average, by 0.72 inseminations (95\% CI: 0.48, 0.95) compared with cows with no mastitis in the same time period. The estimated effect sizes for FSP/AI suggest a risk ratio of conceiving at first insemination of 0.90 (95\% CI: $0.82,0.99)$ for cows with mastitis diagnosed pre-first insemination with respect to cows with no mastitis in the same time period, and a risk ratio of 0.79 (95\% CI: $0.72,0.86)$ for cows diagnosed with mastitis either preor post-first insemination with respect to cows with no mastitis in the same time period. Publication bias was identified in 4 of the meta-analysis models (TFS, DO, SPC, and FSCR), but no influential trials were identified in any models; the reliability of the meta-analysis results should be interpreted carefully keeping these limitations in mind. Further meta-regression analysis would be valuable as additional studies are published that report other potential sources of heterogeneity. Key words: mastitis, pregnancy, meta-analysis

\section{INTRODUCTION}

Understanding what factors contribute to a cow's ability to initiate and maintain pregnancy is crucial for improving reproductive performance and increasing dairy farm profitability. A possible relationship between mastitis incidence and decreased reproductive performance was first reported in Holstein cattle in the early 1990s (Moore et al., 1991). Since then, numerous studies have investigated the relationship between either clinical or subclinical mastitis and reproductive performance.

Most of the studies that have evaluated the effect of mastitis on reproductive outcomes have been observational. One disadvantage of observational studies is that the ability to make overall causal conclusions about an entire population or industry is limited, especially when studies do not agree on outcomes. Literature reviews serve as a qualitative method for summarizing multiple studies conducted on the same topic. Although these reviews do not quantitatively combine research findings 
from multiple sources, they allow an initial qualitative assessment of the body of evidence available to answer a particular question. For example, in a recent publication, Dahl et al. (2017) conducted a systematic literature review to explore the effects of mastitis on pregnancy loss, concluding that the significant effect of mastitis on pregnancy loss was inconsistent between studies. Those authors suggested that further research is needed to better characterize the effects of mastitis not only on pregnancy loss but also on the overall reproductive efficiency of dairy farms with particular attention to characterizing the exposure factors affecting both mastitis and reproduction (Dahl et al., 2017).

One alternative to qualitative literature reviews are quantitative reviews in the form of meta-analysis (Lean et al., 2009; Viechtbauer, 2010). Meta-analysis can provide objective evidence to determine if a relationship exists and to what extent by combining data from multiple studies. It has been almost 2 decades since Fourichon et al. (2000) attempted to summarize the effects of mastitis on the reproductive performance of dairy cows via meta-analysis. That study focused on the effect of several diseases on the reproductive performance of dairy cows with no attempt to quantify and characterize factors that affect those relationships using metaregression. No significant effects of mastitis on many of the examined reproductive performance outcomes were identified, likely because only 9 studies were included in their analysis. A variety of new evidence surrounding this relationship has been published since that study was conducted; however, no new meta-analyses have been conducted to the authors' knowledge.

The objective of this study was to conduct a metaanalysis to quantitatively characterize the effects of mastitis on reproductive performance as well as to identify factors that interact with this relationship. By quantifying and comprehensively characterizing these relationships, producers can better understand how mastitis affects reproductive performance and respond by implementing either strategic breeding protocols to optimize fertility in dairy cows that have experienced mastitis or preventive strategies to reduce mastitis incidence while optimizing the success of future inseminations.

\section{MATERIALS AND METHODS}

\section{Literature Search and Publication Selection}

A systematic literature search for studies that examined the relationship between mastitis incidence and reproductive performance was conducted using the Preferred Reporting Items for Systematic Reviews and
Meta-Analysis Protocols method (Liberati et al., 2009; Moher et al., 2009). On January 26, 2018, 4 online databases were searched: ScienceDirect (Elsevier B.V., Amsterdam, the Netherlands), CAB Direct (CAB International, Wallingford, UK), PubMed (US National Library of Medicine, Bethesda, MD), and Web of Science (Thomson Reuters Science, New York, NY). In all databases, the exact phrase, "mastitis, pregnancy, dairy, cow, cattle" was entered; no publication date or language filters were applied. Results included 2,555, 864, 435, and 148 items from ScienceDirect, PubMed, CAB Direct, and Web of Science, respectively.

All results were screened in 3 steps by (1) title, (2) abstract, and (3) full text to determine eligibility for the meta-analysis. During the first step, publication titles were evaluated for relevance to our research objective. Titles from all 4 databases, or abstracts in cases where titles did not provide enough information, that indicated that the publication evaluated the effects of naturally occurring mastitis on any measure of reproductive performance in dairy cattle were selected for further review $(\mathrm{n}=196)$. After duplicate removal, 126 publications remained. At this point, the titles of all cited works in those 126 publications were reviewed to identify other potentially relevant publications; 71 additional potential publications were identified. All 197 publications were further reviewed by reading the full abstract to confirm if the analysis compared reproductive performance between groups of dairy cows with and without mastitis. Additionally, publications were removed at this point if they were not published in English or if they were not peer-reviewed original research. Thirteen publications were removed because they were published in a language other than English, 22 publications were removed because they were not peer-reviewed original research (i.e., reviews, metaanalyses, non-peer-reviewed conference proceedings, or book chapters), 3 publications were removed because they were copies of another publication (i.e., a conference proceeding and article on the same study), and 93 publications were removed because they did not quantitatively report how mastitis incidence affected reproductive performance outcomes of dairy cattle. Total, 66 publications were identified that appeared relevant to the proposed meta-analysis after abstract evaluation.

Last, a detailed full text evaluation was conducted to determine final eligibility for the meta-analysis based on study characteristics. Study characteristics were evaluated using the PICOS approach (P: population; I: intervention (or exposure) of interest; $\mathrm{C}$ : comparators; O: outcome; and S: study designs; Liberati et al., 2009; Moher et al., 2009). To remain in the meta-analysis, the following study characteristics were required: 
- population: dairy cows of any breed and any parity from dairy herds in any country;

- exposure of interest: clinical or subclinical mastitis where clinical mastitis indicated detection of abnormal milk, abnormal udder characteristics, or recorded treatment and subclinical mastitis was identified by SCC evaluation or culture results;

- comparators: dairy cows that had not been diagnosed with clinical or subclinical mastitis;

- outcome of interest: reproductive performance, as measured by any common dairy cow reproduction performance metric; and

- study designs: any.

The outcome of interest was intentionally left broad because of uncertainty about how most publications would report differences in reproductive performance. During full text evaluation, it became apparent that some of the publications reported multiple effects from different trials (i.e., comparing cows getting mastitis at different time points or different severity levels to the same control group). Therefore, a minimum of 10 trials for any reproductive performance outcome was arbitrarily set as the requirement for inclusion of that outcome as a response variable in the meta-analysis. The reproductive performance outcomes meeting this criteria were time to first service (TFS), days open (DO), services per conception (SPC), pregnancies per insemination at first service (FSP / AI), and pregnancy loss (PL). In all trials, TFS and DO were measured as the average number of days from calving until the event of interest (first service or conception), and although not explicitly stated in all studies, both outcomes appeared to always include the voluntary waiting period and DO appeared to only include cows that became pregnant. Services per conception was measured as the average number of services per pregnancy in pregnant cows. Although both SPC and DO are common reproductive metrics in the dairy industry, it is important to point out that by only including pregnant cows in these measurements, the effect of mastitis on replacement dynamics (i.e., culling) is not accounted for. Therefore, the resulting measurements do not reflect the fact that cows experiencing mastitis are more likely to be culled than healthy cows. Pregnancies per insemination at first service was measured as the number of FSP/AI. The definition used for PL varied by trial. Some trials considered PL occurring only in early gestation (35 to $75 \mathrm{~d}$ pregnant, $\mathrm{n}=6$ trials), whereas other studies considered PL occurring at any time during gestation ( $\mathrm{n}=7$ trials). Because of the limited number of trials available in that analysis, all results were combined into the same analysis, regardless of PL definition.
Although the criteria for study design type was left broad, only observational studies were available for use in this meta-analysis. Controversy exists over the use of observational studies in meta-analyses (Shrier et al., 2007), but the only available randomized controlled trials evaluating how mastitis affects reproductive performance would be challenge studies where mastitis was induced. In our case, we were interested in the effects of naturally occurring mastitis. After full text review, 51 publications remained eligible for inclusion in the meta-analysis.

\section{Data Extraction}

Information from the eligible publications was compiled using an electronic form created in Microsoft Excel (Microsoft Corp., Redmond, WA). Extracted study characteristics included (1) year of publication, (2) country of the study (assumed to be the location of the first author if not explicitly stated), (3) breed of cattle, (4) mastitis definition, and (5) mastitis timing relative to first insemination and relative to the insemination leading to conception. Extracted outcome data included (1) the type(s) of outcome(s) reported (TFS, DO, SPC, FSP/AI, or PL; many studies reported more than 1 outcome), (2) the number of cows classified as controls or exposed to mastitis, and (3) the outcome for each group of cows (Appendix Tables A1, A2, A3, A4, and A5). The outcome could have been the mean and associated standard deviation (SD), the proportion of animals experiencing an event, or the odds ratio and associated 95\% confidence interval (CI) for the outcome of interest. When the standard error (SE) or standard error of the mean was reported instead of the SD, the SD was back-calculated by multiplying the standard error of the mean or SE by the square root of the group (control or mastitis) sample size. In one study (Nguyen et al., 2011), uncertainty was labeled as the SE but was determined to be the SD after correspondence with the authors and was therefore not back-calculated. The type of uncertainty reported was not defined in 2 studies. After correspondence with the authors, the reported uncertainty in Schrick et al. (2001) was determined to be the SEM. For Smulski et al. (2006), the corresponding author's email address was no longer active so uncertainty was assumed to represent the SD based on the values of the uncertainties relative to the reported mean. Through the data extraction process, multiple publications were identified that did not provide adequate information on the number of animals in each group or the outcome of interest to calculate effect size; those publications were excluded 
from the meta-analysis. In total, 29 publications were included in the meta-analysis.

\section{Statistical Analysis}

All statistical analyses were performed using the metafor package (Viechtbauer, 2010) in $\mathrm{R}$ version 3.4.2 (R Foundation for Statistical Computing, Vienna, Austria). Separate meta-analyses were conducted for each reproductive performance parameter (TFS, DO, SPC, FSP/AI, and PL), for a total of 5 meta-analyses. In standard meta-analytic models, independence between each effect size estimate is assumed (Viechtbauer, 2010). Because we included results from multiple trials within a single publication, multivariate, multilevel linear mixed-effects models (rma.mv function in metafor) were chosen for use in this meta-analysis, as described in Viechtbauer (2010). In short, these models account for the non-independence of the effect sizes analyzed in the meta-analysis by including a variance-covariance matrix of the sampling errors in place of a vector of the sampling variances of independent effect sizes.

All multilevel models were fitted via the maximumlikelihood estimation methods, including publication as a random effect. Initially, trial within publication was also included in the model as an additional random effect, but its inclusion in the model caused the estimation of zero variance components for many of the models so the decision was made to reduce the random effects structure of the model, maintaining only a random effect for publication. The mean difference between mastitis exposed and control cows was chosen as the effect size for TFS, DO, and SPC because of its ease of interpretation. The log risk ratio was chosen as the effect size for binomial response variables (FSP/AI and PL) and results were back-transformed to represent risk ratios. Risk ratios are calculated as the incidence of interest (i.e., conception for FSP/AI and pregnancy loss for PL) in an exposed group divided by the incidence of interest in an unexposed group. In this study, exposure would represent mastitis occurrence. All effect sizes were calculated using the escalc() function in the metafor package when data were available. In some studies that evaluated PL, only odds ratios or risk ratios and 95\% CI were reported rather than the proportion observed in both the exposed and control groups of cows. In those cases, the effect size was calculated as the log of the risk ratio (odds ratios were converted to risk ratios by rebuilding contingency tables, if necessary) and the sampling variance was calculated using the CI. Effect sizes were weighted using a weight matrix created with the inverse of the variance-covariance matrix as described in Viechtbauer (2010).

\section{Heterogeneity and Meta-Regression}

After estimating the pooled effect sizes, the $\mathrm{I}^{2}$ statistic was calculated for each model. The $\mathrm{I}^{2}$ statistic represents the percent of the total variance in the model that can be attributed to between study heterogeneity rather than to random chance (Higgins and Thompson, 2002). The Cochrane Q statistic was evaluated to determine heterogeneity significance. For models where heterogeneity was significant, meta-regression analysis was conducted to explore the sources of heterogeneity. It is important to point out that moderator inclusion depended on the availability of data in each data set. The only moderator evaluated consistently in all models was the country of the study (classified as the United States or other). Models for TFS, DO, and SPC also included the breed of the majority of the cows used in the study (classified as Holstein or other) as a moderator; the remaining models did not include enough observations from publications evaluating nonHolsteins to include this as a moderator.

Mastitis type (clinical or subclinical) was considered as a moderator in the models for DO and FSP/AI; the remaining models did not include enough observations from publications evaluating subclinical mastitis to include this as a moderator. Specifically, the models for both TFS and SPC each only included 2 trials that evaluated subclinical mastitis. Although insufficient for inclusion as a moderator in the meta-regression models, the pooled effect size estimate was calculated both with and without subclinical mastitis trial inclusion, and because only minimal differences were found (data not shown), the decision was made to retain the subclinical mastitis case trials in both models for pooled effect size estimates. Important to note is that subclinical mastitis was defined differently in different studies. All trials that evaluated subclinical mastitis conducted monthly testing to evaluate subclinical mastitis status, except Schrick et al. (2001) who tested cows every 4 to 8 wk. To define subclinical mastitis, various definitions were used. Both Lomander et al. (2013) and McNally et al. (2014) used the cutoff of $>200,000$ cells/mL to indicate infection, whereas Fuenzalida et al. (2015) used a stricter cutoff of $>150,000$ cells $/ \mathrm{mL}$ and Nguyen et al. (2011) defined 4 different subclinical mastitis categories: 50,000 to 100,000 cells/mL, 100,000 to 200,000 cells $/ \mathrm{mL}, 200,000$ to 500,000 cells $/ \mathrm{mL}$, and $>500,000$ cells $/ \mathrm{mL}$ (all of which were included in our analysis as separate trials). Alternatively, Jousan et al. (2005) used somatic cell count score to define subclinical mastitis in 2 groups: either somatic cell count score between 2.5 and 5 or $>5.0$. Finally, Schrick et al. (2001) cultured all milk samples and defined subclinical mastitis as the 
presence of the same pathogen in at least 2 consecutive samples without clinical mastitis occurrence.

Mastitis diagnosis timing was considered as a moderator in all meta-regression models, but was classified differently for different outcomes. For TFS and FSP/ AI, mastitis diagnosis timing was classified as pre-, post-, or either pre- or post-first insemination (i.e., cows diagnosed with mastitis either before or after first insemination were grouped together in some studies); the exact DIM that these categories included varied by trial. Although the logic behind these diagnosis timing classifications may not always be intuitive (i.e., mastitis occurring post-first insemination is not expected to influence TFS), these diagnosis timing categories were chosen based on how the trials included in the meta-analysis had differentiated mastitis diagnosis timing for their statistical analysis. Additionally, diagnosis timing may not accurately represent actual onset of disease. Therefore, diagnosis post-first insemination could represent a case of mastitis that began before first insemination but was not identified until after first insemination. For DO and SPC, mastitis diagnosis timing was classified as pre- or either pre- or postinsemination leading to conception (i.e., cows classified as diagnosed with mastitis only after or cows classified as diagnosed with mastitis either before or after the insemination leading to conception were grouped together in the latter category); again, the exact DIM that these categories included varied by trial. Other moderators that were considered for inclusion but were not evaluated because of limited reporting by trials included milk production level, parity group, breeding method (i.e., synchronization vs. estrus detection), season of breeding, housing type, bedding type, mean BCS, occurrence of other diseases, mastitis treatment, and the bacteriological cause of mastitis.

Before conducting meta-regression analysis, available moderators for each model were evaluated for multicollinearity by calculating the variance inflation factors; all variance inflation factor measures were $<10$. The first step in our meta-regression analysis was to evaluate each full meta-regression model with all moderator variables in the model. The $\mathrm{I}^{2}$ statistic was calculated for all full models to assess how inclusion of the selected moderators changed heterogeneity relative to the models without moderators. Next, a model selection strategy was implemented with the fitting of each model with all possible combinations of individual moderators; no interactions between moderators were tested. The model with the lowest Akaike information criterion was selected as the final model. Within the final models, moderator significance was assumed when $P<0.05$. The $\mathrm{I}^{2}$ statistic was again calculated for each meta-regression model after moderator reduction to evaluate the change in the amount of total variance attributed to heterogeneity.

\section{Publication Bias}

Asymmetry of study publication was evaluated by including the sampling variance of the effect size as a moderator in the final meta-regression models to conduct Egger's regression test (Egger et al., 1997). If the intercept of the regression test significantly deviated from zero, bias was considered present. For this test, the significance level was set at $P<0.05$ and significant results were visualized by graphing individual trial variance against the trial effect size, commonly known as funnel plots.

\section{Identification of Influential Trials}

The sensitivity of the pooled effect size estimates to influential trials was tested using a leave-one-out approach. For each model, the pooled effect size estimate of the multilevel model without moderators was recalculated for all combinations of trials, leaving one trial out at a time. In all leave-one-out analyses, a trial was considered influential if its removal changed the direction or significance of the pooled effect size estimate.

\section{RESULTS}

The trials selected for the meta-analysis of TFS, DO, SPC, FSP/AI, and PL are summarized in Table 1. The estimated pooled effect sizes for each reproductive performance variable obtained with the random effects meta-analytic models, not including moderator variables, are included in Table 2 . The effect sizes, estimated through meta-regression models that contained moderators, are also presented in Table 2. On average, across all analyzed trials, mastitis incidence significantly increased TFS, DO, SPC, and PL, while decreasing FSP/AI. Forest plots showing the effect of mastitis incidence on each reproductive performance are presented for all outcomes. When meta-regression models, with moderator variables, were the final models used to represent the outcomes, forest plots were constructed with the meta-regression models (Figures 1, 2, 3, and 4). Conversely, when meta-regression models were not used as final models, forest plots were constructed using random effects models (Figure 5). For Figures 1, 2,3 , and 4 , the corresponding forest plots associated with the random effects models are presented in the Appendix (Figures A1 to A4).

The $\mathrm{I}^{2}$ statistics for the TFS, DO, SPC, FSP/AI, and PL models without moderators were 94.9, 89.6, 47.1, 92.9 , and $<1.0 \%$, respectively. Heterogeneity, based on 


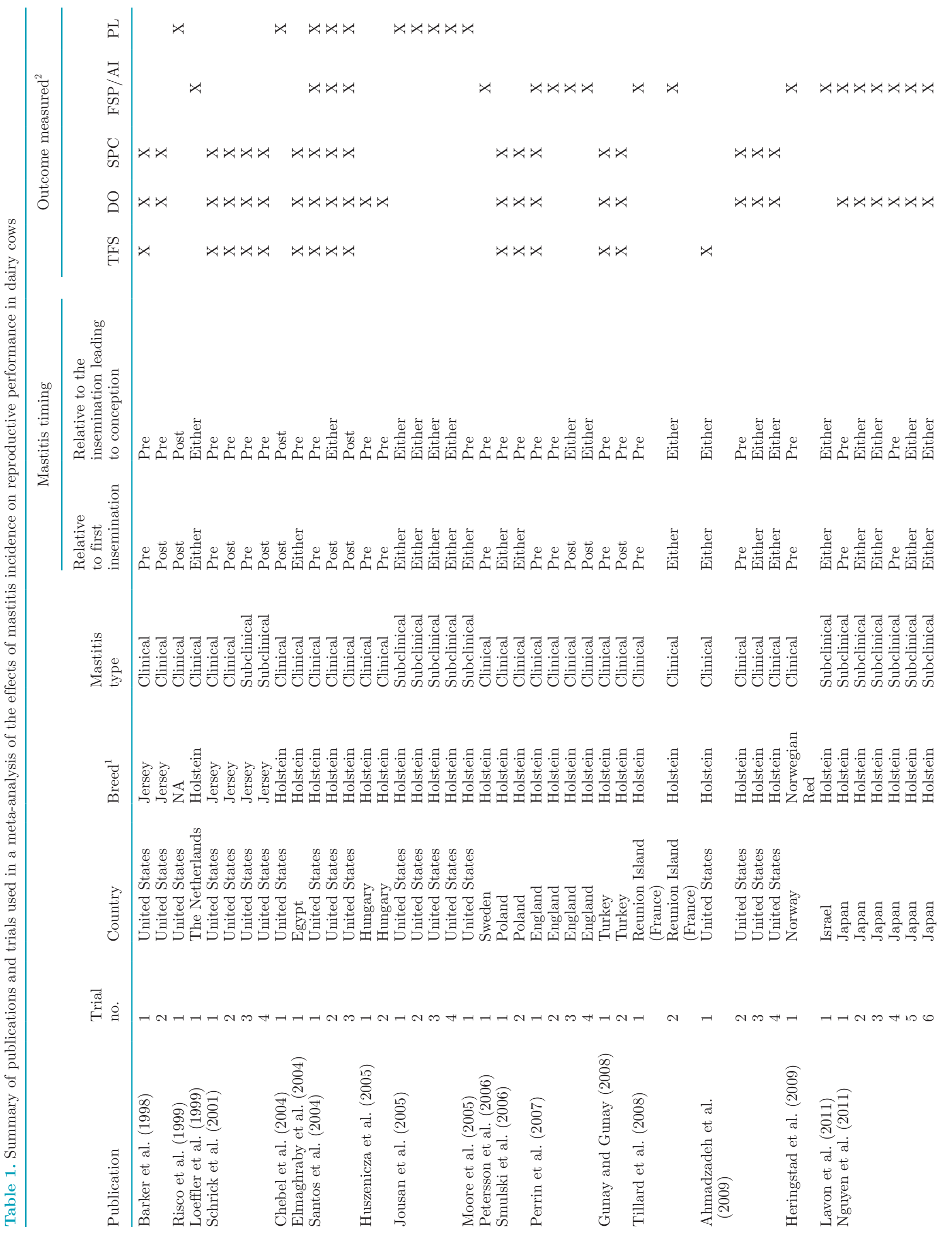


DOLECHECK ET AL.

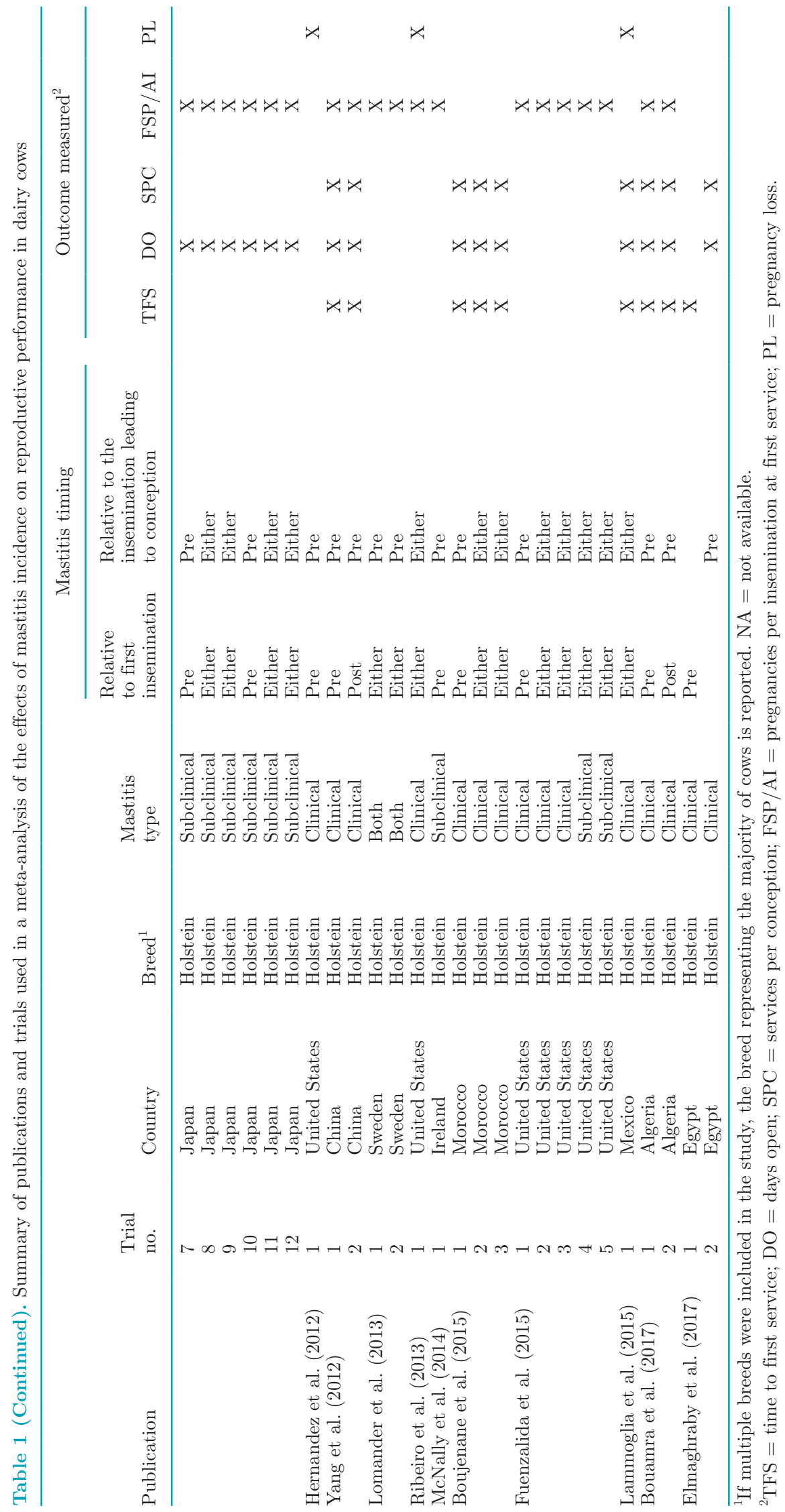


the Cochrane's Q statistic, was present in all analyses except for the analysis of PL. Because heterogeneity was not significant in the PL model, meta-regression was not conducted and the random effects model was considered the final PL model. However, it is important to point out that the between-publication variance for this model was estimated to be 0 , likely because of the small number of publications $(n=8)$ included in the analysis for this outcome (Chung et al., 2013). The $\mathrm{I}^{2}$ statistics for the full TFS, DO, SPC, and FSP/AI models (i.e., with all moderators included) were 92.9, $89.3,44.9$, and $88.2 \%$, respectively. The relatively small changes in the $\mathrm{I}^{2}$ statistic when all moderators were included in the models compared with the models with no moderators indicates that additional moderators beyond those explored in this study may be valuable to explain more of the heterogeneity among publications.

Mastitis type was a significant moderator in the meta-regression model for DO; studies measuring clinical mastitis found a larger mean difference between cows with and without mastitis than studies measuring subclinical mastitis. Mastitis diagnosis timing was a significant moderator in the meta-regression models for TFS,
DO, SPC, and FSP/AI. When mastitis was diagnosed at post-first insemination or either pre- or post-first insemination, the mean difference in TFS between cows with and without mastitis was not significantly different than zero. However, when mastitis was diagnosed pre-first insemination, cows with mastitis had a greater TFS than cows without mastitis. When subclinical mastitis was diagnosed pre-insemination leading to conception, the mean difference in DO between cows with and without subclinical mastitis was not significantly different than zero. However, when subclinical mastitis was diagnosed either pre- or post-insemination leading to conception, cows with mastitis experienced a mean increase in DO compared with cows without mastitis. Regardless of when clinical mastitis was diagnosed (pre-insemination leading to conception or either preor post-insemination leading to conception), the mean DO increased compared with cows without mastitis. The mean difference between cows with mastitis and cows without mastitis for the average SPC was smaller when mastitis was diagnosed at pre-insemination leading to conception compared with cows when mastitis was diagnosed either pre- or post-insemination leading

Table 2. Effect size estimates of mastitis incidence on reproductive performance outcomes in dairy cows derived both with random-effects multilevel meta-analysis models (pooled effect sizes) and the meta-regression models

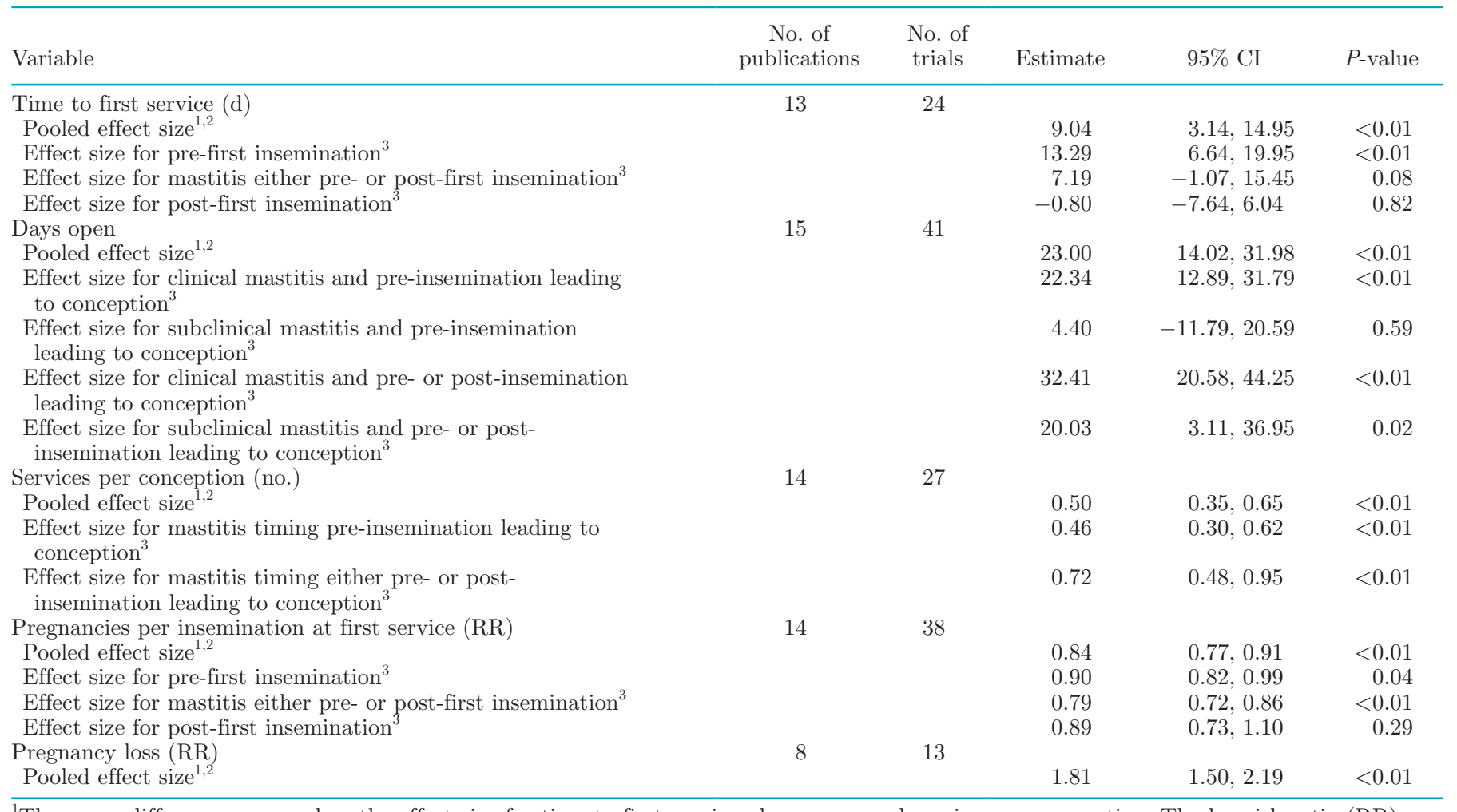

${ }^{1}$ The mean difference was used as the effect size for time to first service, days open, and services per conception. The log risk ratio (RR) was used as the effect size for pregnancies per insemination at first service and pregnancy loss; outcomes were back-transformed to represent RR.

${ }^{2}$ Pooled effect sizes estimated by random effects models, not adjusted by moderator variables.

${ }^{3}$ Predicted using the estimates of intercept and factor level effects from meta-regression models. 
to conception. Finally, the log-risk ratio for FSP/AI was significantly different than zero when mastitis occurred pre-first insemination or either pre- or post-first insemination. The $\mathrm{I}^{2}$ statistics for the TFS, DO, SPC, and FSP/AI models after moderator selection (i.e., the final models) all remained similar to the full models (93.8, 90.2, 53.1, and 88.8\%, respectively).

The Egger's test for publication bias was not significant for the PL model $(P=0.14)$, but was significant for TFS $(P=0.01)$, DO $(P<0.01)$, SPC $(P<0.01)$, and FSP/AI $(P<0.01)$, indicating that publication bias was present for these outcomes. The relationships between the effect sizes and the sampling variances are presented in Figure 6. The leave-one-out evaluation for influential trials demonstrated that removal of any individual trial effect sizes did not change the direction or significance of the pooled effect size estimates for all analyses (TFS, DO, SPC, FSP/AI, and PL). After all analyses, the final models for TFS, DO, SPC, and FSP/AI were the meta-regression models, whereas the final model for PL was the pooled effect size model. Forest plots from each final model are presented as Figures 1 to 5 .

\section{DISCUSSION}

To the authors' knowledge, the last meta-analysis that evaluated how mastitis incidence affects reproductive performance was conducted in 2000 by Fourichon et al. In their analysis of 9 studies, mastitis incidence was found to affect occurrence of insemination [relative risk $(95 \% \mathrm{CI})=0.74(0.69,0.79)]$ and to have minimal effects on days to first service [days $(95 \% \mathrm{CI})=0.8$ $(0.2,1.5)]$ and pregnancies per insemination from 45 to 184 d postpartum [hazard ratio $(95 \%$ CI $)=0.80(0.67$, 0.96)]. No effect of mastitis incidence on FSP/AI, the interval from first service to conception, DO, pregnancies per insemination from 56 to $120 \mathrm{~d}$ postpartum, or $\mathrm{SPC}$ was found. Overall, the authors concluded that mastitis had no significant effect on reproduction in

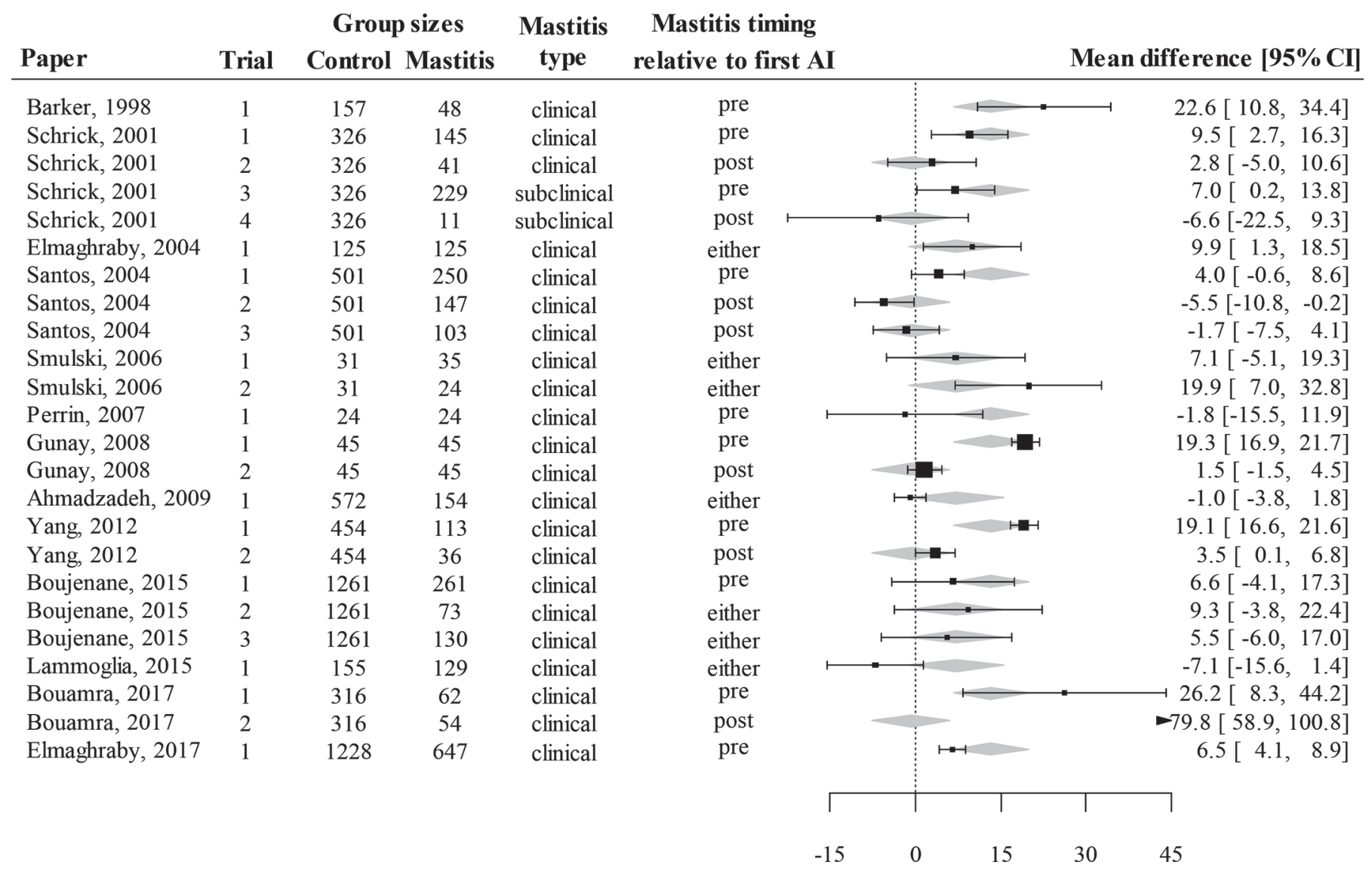

Figure 1. Forest plot to evaluate the effect of mastitis incidence on time to first service in dairy cows. The effect size for each individual trial is reported and the size of the box for each trial indicates its weight in the pooled estimate. Mastitis incidence could have been clinical (identified by detection of abnormal milk, abnormal udder characteristics, or recorded treatment) or subclinical (identified by SCC evaluation or culture results). The gray polygons represent the predicted effect sizes using the meta-regression model. Papers are listed by first author and year of publication. 


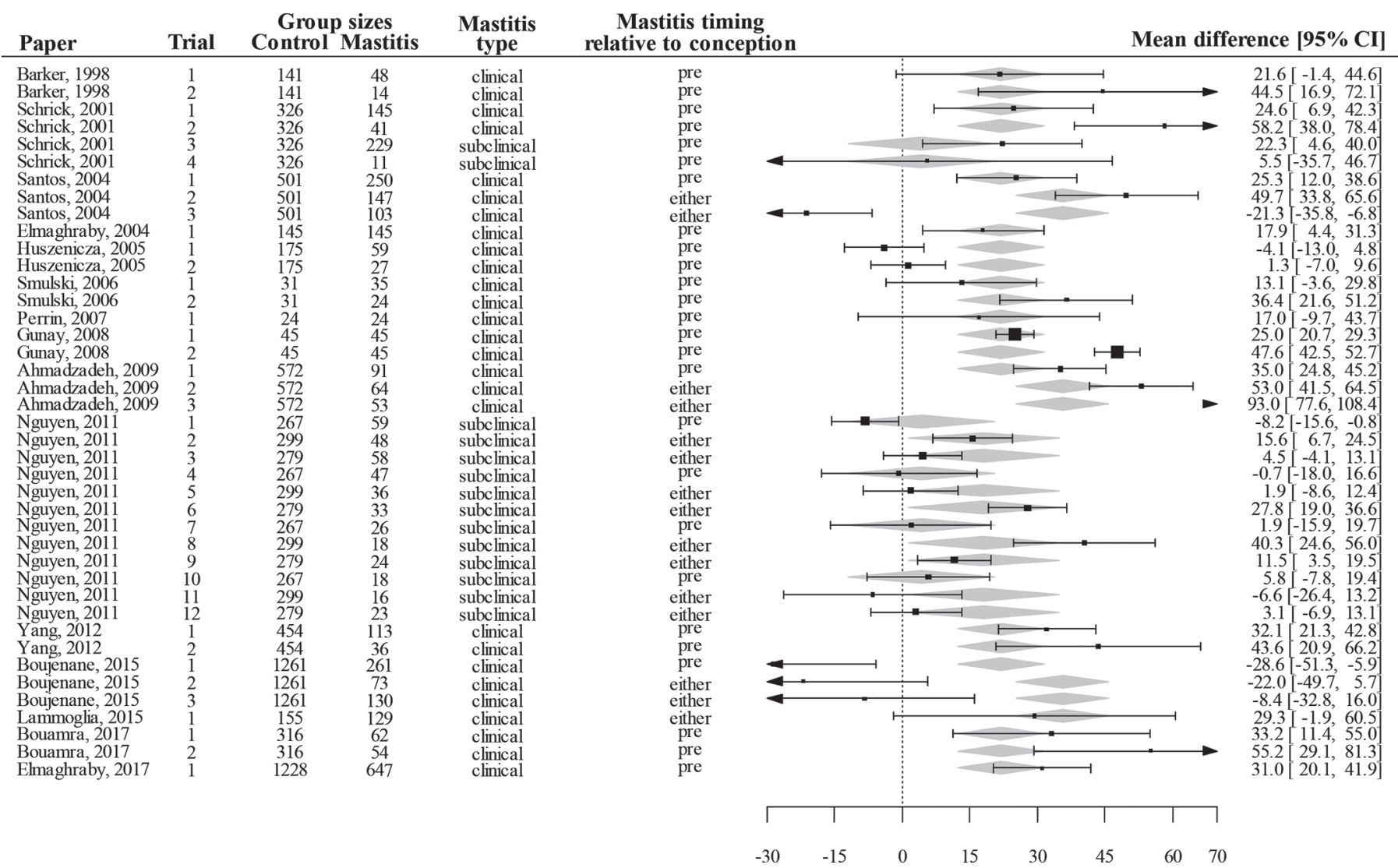

Figure 2. Forest plot to evaluate the effect of mastitis incidence on days open in dairy cows. The effect size for each individual trial is reported and the size of the box for each trial indicates its weight in the pooled estimate. Mastitis incidence could have been clinical (identified by detection of abnormal milk, abnormal udder characteristics, or recorded treatment) or subclinical (identified by SCC evaluation or culture results). The gray polygons represent the predicted effect sizes using the meta-regression model. Papers are listed by first author and year of publication.

dairy cattle. Since that study was published, multiple additional studies have evaluated the relationship between mastitis incidence and reproductive performance. The number of publications that were available for the current meta-analysis $(\mathrm{n}=29)$ was over 3 times the number used by Fourichon et al. (2000), with many publications including results from multiple trials. Additionally, the higher producing cows evaluated in more recent studies may be more susceptible to mastitis (Fleischer et al., 2001).

The pooled effect sizes estimated with random effects models for TFS, DO, and SPC reported in this metaanalysis can be directly interpreted as the expected difference in reproductive performance between cows that experience mastitis in the current lactation and cows that do not experience mastitis. For example, considering the pooled estimates from the random effects models in Table 2, cows with mastitis would, on average, be expected to require 9.04 (95\% CI: 3.14 to 14.95) additional days before first service, 23.0 (95\%
CI: 14.02 to 31.98$)$ additional days to pregnancy, and 0.50 (95\% CI: 0.35 to 0.65 ) additional SPC compared with cows that do not experience mastitis. Similarly, the pooled effect sizes for FSP/AI and PL, as estimated by random effects models, are directly interpretable as the ratio in risk between cows that do and do not experience mastitis in the current lactation, in the average situation. Therefore, cows that experience mastitis have, on average, 0.84 (95\% CI: 0.77 to 0.91 ) times the risk of conceiving at first service and 1.81 (95\% CI: 1.50 to 2.19) times the risk of PL during gestation as cows that do not experience mastitis. All of the pooled effect sizes in this meta-analysis were significantly different than zero; however, large CI around the estimates caution that large variations in the reproductive performance response of individual herds and animals to mastitis incidence could be observed. Additionally, the substantially large heterogeneity in the models of most reproductive outcomes suggests that although predictions on future herds will be, on average, appropriate, 
predictions for a specific future herd will likely suffer from large variance.

The mechanism behind the relationship between mastitis and reproductive performance, however, remains to be fully understood. For instance, in studies considering cows that developed naturally occurring mastitis, researchers have found delayed ovulation, low estradiol levels, abnormal preovulatory LH surges, and atypical folliculogenesis (Lavon et al., 2010; Rahman et al., 2012). These irregularities could lead to reduced estrus expression and reduced fertilization success after insemination. Additionally, the immune response associated with mastitis leads to the production of pro-inflammatory cytokines that, in combination with endotoxins produced from the bacteria causing mastitis, can disrupt normal hormone levels, negatively affect corpus luteum development, and impair embryonic development (Soto et al., 2003; Hansen et al., 2004;
Schams and Berisha, 2004). The immune response also increases body temperature, which, similar to heat stress, could reduce embryonic survival (Hansen et al., 2004). Ultimately, the relationship between mastitis and fertility is multifaceted.

\section{Heterogeneity}

Four of the 5 meta-analysis conducted in this study included substantial between-publication heterogeneity. Overall, an exploration of heterogeneity by including moderators within each model helped to explain only minimal variation, as evaluated by the $\mathrm{I}^{2}$ statistics. Two moderators that we, a priori, expected to affect the relationship between mastitis incidence and reproductive performance were the type and timing of mastitis. Parameter estimates from final meta-regression models can be used to quantify the effect of moderator

\begin{tabular}{|c|c|c|c|c|c|c|c|}
\hline \multirow{2}{*}{$\begin{array}{l}\text { Publication } \\
\text { Barker, } 1998\end{array}$} & \multirow{2}{*}{$\begin{array}{c}\text { Trial } \\
1\end{array}$} & \multicolumn{2}{|c|}{$\begin{array}{c}\text { Group sizes } \\
\text { Control Mastitis } \\
\end{array}$} & \multirow{2}{*}{$\begin{array}{c}\begin{array}{c}\text { Mastitis } \\
\text { type }\end{array} \\
\text { clinical }\end{array}$} & \multicolumn{2}{|c|}{$\begin{array}{l}\text { Mastitis timing } \\
\text { relative to conception }\end{array}$} & $\begin{array}{r}\text { Mean difference } \\
{[95 \% \mathrm{CI}]}\end{array}$ \\
\hline & & 143 & 46 & & pre & $\rightarrow$ & $-0.1[-0.7,0.5]$ \\
\hline Barker, 1998 & 2 & 143 & 13 & clinical & pre & $\longmapsto$ & $1.2[0.6,1.8]$ \\
\hline Schrick, 2001 & 1 & 326 & 145 & clinical & pre & $\longrightarrow$ & $0.5[-0.1,1.1]$ \\
\hline Schrick, 2001 & 2 & 326 & 41 & clinical & pre & $\rightarrow \longmapsto$ & $1.4[0.8,2.0]$ \\
\hline Schrick, 2001 & 3 & 326 & 229 & subclinical & pre & $\longrightarrow$ & $0.5[-0.1,1.1]$ \\
\hline Schrick, 2001 & 4 & 326 & 11 & subclinical & pre & & $0.5[-0.6,1.6]$ \\
\hline Santos, 2004 & 1 & 501 & 250 & clinical & pre & $\longmapsto$ & $0.0[-0.3,0.4]$ \\
\hline Santos, 2004 & 2 & 501 & 147 & clinical & either & $\longmapsto$ & $0.5[0.0,0.9]$ \\
\hline Santos, 2004 & 3 & 501 & 103 & clinical & either & $\longmapsto$ & $-0.1[-0.5,0.3]$ \\
\hline Elmaghraby, 2004 & 1 & 145 & 145 & clinical & pre & $\rightarrow$ & $0.3[-0.0,0.7]$ \\
\hline Smulski, 2006 & 1 & 31 & 35 & clinical & pre & $b=1$ & $0.3[-0.0,0.6]$ \\
\hline Smulski, 2006 & 2 & 31 & 24 & clinical & pre & 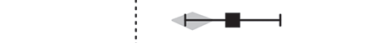 & $0.8[0.4,1.2]$ \\
\hline Perrin, 2007 & 1 & 24 & 24 & clinical & pre & $\longmapsto$ & $0.3[-0.3,1.0]$ \\
\hline Gunay, 2008 & 1 & 45 & 45 & clinical & pre & $=-1$ & $0.3[-0.1,0.7]$ \\
\hline Gunay, 2008 & 2 & 45 & 45 & clinical & pre & $\longmapsto$ & $1.6[1.2,2.0]$ \\
\hline Ahmadzadeh, 2009 & 91 & 572 & 91 & clinical & pre & $\mapsto$ & $0.4[0.1,0.6]$ \\
\hline Ahmadzadeh, 2009 & 92 & 572 & 64 & clinical & either & $\longmapsto-1$ & $0.7[0.4,1.0]$ \\
\hline Ahmadzadeh, 2009 & 93 & 572 & 53 & clinical & either & $\infty \longmapsto$ & $1.5[1.1,1.9]$ \\
\hline Yang, 2012 & 1 & 454 & 113 & clinical & pre & b & $0.3[0.2,0.5]$ \\
\hline Yang, 2012 & 2 & 454 & 36 & clinical & pre & $\Leftrightarrow-1$ & $0.7[0.3,1.0]$ \\
\hline Boujenane, 2015 & 1 & 1261 & 261 & clinical & pre & -1 & $-0.5[-1.7,0.8]$ \\
\hline Boujenane, 2015 & 2 & 1261 & 73 & clinical & either & $\Longrightarrow$ & $-0.3[-1.6,0.9]$ \\
\hline Boujenane, 2015 & 3 & 1261 & 130 & clinical & either & $\longrightarrow$ & $-0.3[-1.5,1.0]$ \\
\hline Lammoglia, 2015 & 1 & 155 & 129 & clinical & either & $\rightarrow$ & $0.7[-0.1,1.5]$ \\
\hline Bouamra, 2017 & 1 & 316 & 62 & clinical & pre & & $0.5[-1.6,2.6]$ \\
\hline & 2 & 316 & 54 & clinical & pre & $\longrightarrow$ & $0.6[-1.7,2.8]$ \\
\hline \multirow[t]{3}{*}{ Elmaghraby, 2017} & 1 & 1228 & 647 & clinical & pre & $\longmapsto$ & $0.5[0.2,0.8]$ \\
\hline & & & & & & $T$ & \\
\hline & & & & & & -0.50 & 2.5 \\
\hline
\end{tabular}

Figure 3. Forest plot to evaluate the effect of mastitis incidence on services per conception in dairy cows. The effect size for each individual trial is reported and the size of the box for each trial indicates its weight in the pooled estimate. Mastitis incidence could have been clinical (identified by detection of abnormal milk, abnormal udder characteristics, or recorded treatment) or subclinical (identified by SCC evaluation or culture results). The gray polygons represent the effect sizes predicted by the meta-regression models. Papers are listed by first author and year of publication. 
variables on the effect sizes (Table 2). In particular, we used the parameter estimates (i.e., intercept plus estimates of factor level effects or regression coefficients) from the meta-regression models to predict the effect sizes determined by observations in different groups, as determined by the moderators in the model. For instance, the meta-regression models for TFS estimated an effect size for mastitis occurring pre-first insemination of an additional 13.29 d (95\% CI: 6.64, 19.95) when compared with cows with no mastitis in the same time period. On the contrary, the effect size of cows with mastitis either pre- or post-first insemination was estimated to not differ from zero (effect size: 7.19; $95 \%$ CI: $-1.07,15.45 ; P$-value $=0.08)$ in relation to cows with no mastitis at the same time period. Likewise, for cows with mastitis diagnosed post-first insemination, the effect size was not significantly different than zero (effect size: $-0.80 ; 95 \%$ CI: $-7.64,6.04 ; P$-value
$=0.46$ ), suggesting no difference in TFS for cows with mastitis post-first insemination and cows with no mastitis in the same time period.

Furthermore, the meta-regression models for DO suggest that, with relation to cows with no mastitis in the same time period, the mean difference is 22.34 additional DO (95\% CI: 12.89, 31.79) for cows with clinical mastitis diagnosed at pre-insemination leading to conception. For cows with clinical mastitis diagnosed either pre-or post-insemination leading to conception, the estimated mean difference suggested an additional 32.41 DO (95\% CI: $20.58,44.25)$ compared with cows without clinical mastitis in the same time period. For cows with subclinical mastitis diagnosed either pre- or post-insemination, 20.03 additional DO were estimated in comparison with cows with no mastitis in the same time period. The mean difference in DO was not significantly different than zero for cows with subclinical

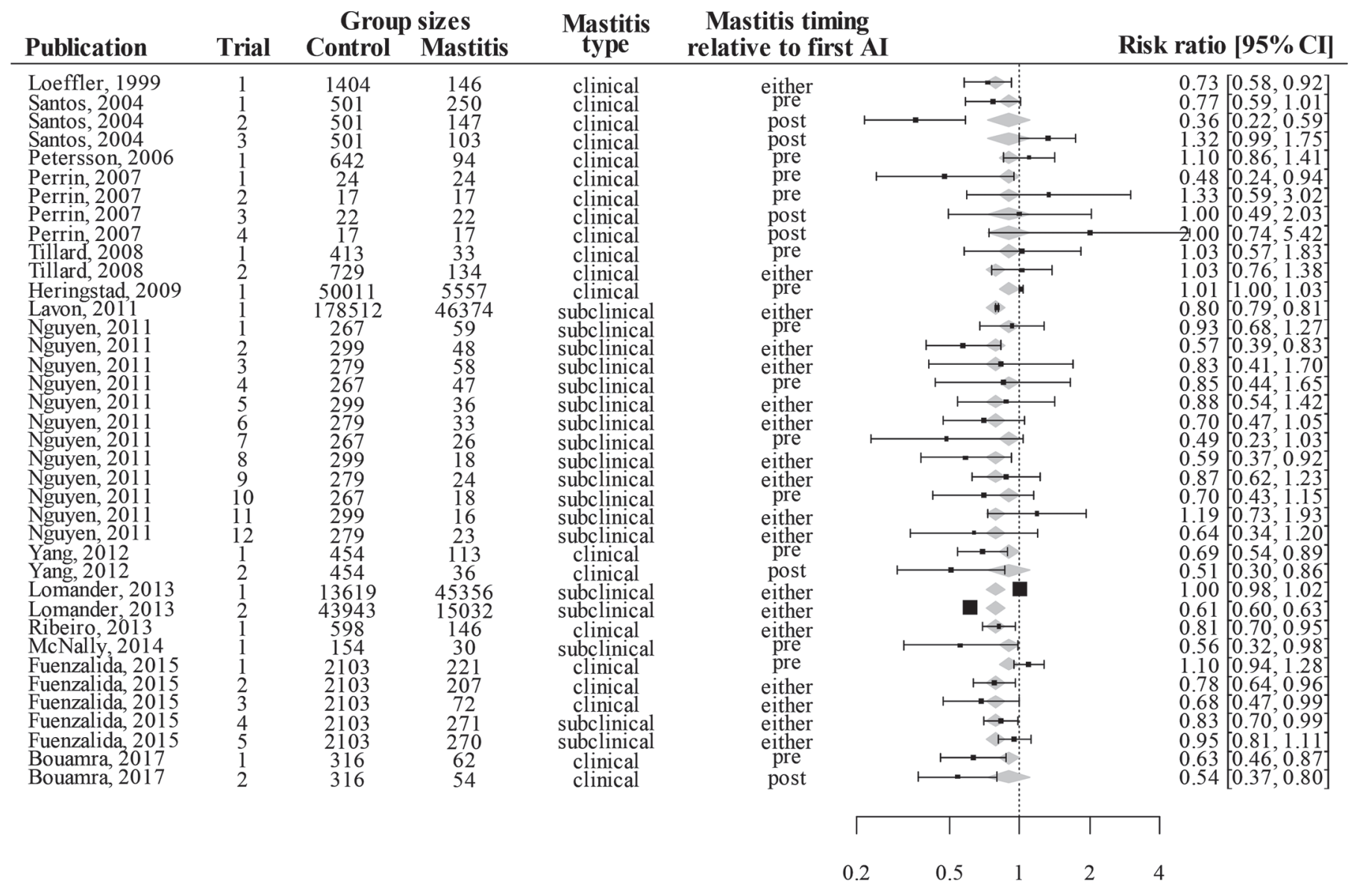

Figure 4. Forest plot to evaluate the effect of mastitis incidence on pregnancies per insemination at first service in dairy cows estimated using random effects models. The effect size for each individual trial is reported and the size of the box for each trial indicates its weight in the pooled estimate. Mastitis incidence could have been clinical (identified by detection of abnormal milk, abnormal udder characteristics, or recorded treatment) or subclinical (identified by SCC evaluation or culture results). The gray polygons represent the effect sizes predicted by the meta-regression models. Papers are listed by first author and year of publication. 
mastitis diagnosed pre-insemination (effect size: 4.40; 95\% CI: $-11.78,20.59 ; P=0.59)$ compared with cows with no mastitis in the same time period.

The effect size estimates from the meta-regression models for SPC suggest that, on average, SPC increases by 0.46 inseminations (95\% CI: $0.30,0.62)$ for cows experiencing mastitis pre-insemination leading to conception compared with cows not experiencing mastitis in the same time period. When mastitis occurred either pre- or post-insemination leading to conception, a cow experiencing mastitis would be expected to have SPC increase by $0.72(95 \%$ CI: $0.48,0.95)$ inseminations compared with a cow without mastitis in the same time period. The estimated effect sizes for FSP/AI suggest a risk ratio of conceiving at first insemination is 0.90 (95\% CI: $0.82,0.99)$ for cows with mastitis diagnosed pre-first insemination and 0.79 (95\% CI: $0.72,0.86)$ for cows diagnosed with mastitis either pre- or post-first insemination when compared with cows with no mastitis in those time periods. The log risk ratio was not different than zero for cows diagnosed with mastitis post-first insemination (effect size: $0.89 ; 95 \%$ CI: 0.73 , $1.10 ; P=0.29)$.

The type of mastitis (clinical or subclinical) could be an indication of severity, which would lead to the hypothesis that clinical mastitis incidence would have a larger negative effect on reproductive performance, as observed in the estimated effect sizes of the meta- regression analysis of DO (Table 2). However, if clinical cases are treated and subclinical cases are not, there might be scenarios where subclinical cases could have similar or even greater negative performance effects on the cow. Ideally, more in depth measures of severity, details about the bacteria causing mastitis and the occurrence and type of mastitis treatment would be included as moderators in the meta-regression. However, this level of detailed information was not consistently available in the studies used in our analysis.

Depending on the exact mechanism of how mastitis affects reproduction, the timing of a case of mastitis relative to follicle development, ovulation, insemination, conception, early embryo development, implantation, or other reproductive events could change how occurrence affects reproductive performance. Based on how most studies reported mastitis occurrence, mastitis diagnosis timing relative to first insemination was assessed for TFS and FSP/AI. Three diagnosis timing categories were included: pre-first insemination, post-first insemination, and either pre- or post-first insemination. Mastitis diagnosis post-first insemination or either pre- or post-first insemination resulted in no significant mean difference for days to first service in support of the hypothesis that for mastitis incidence to increased TFS, it would need to occur before first insemination. Further, the log risk ratio for FSP/AI was not different than zero for cows with mastitis diagnosed

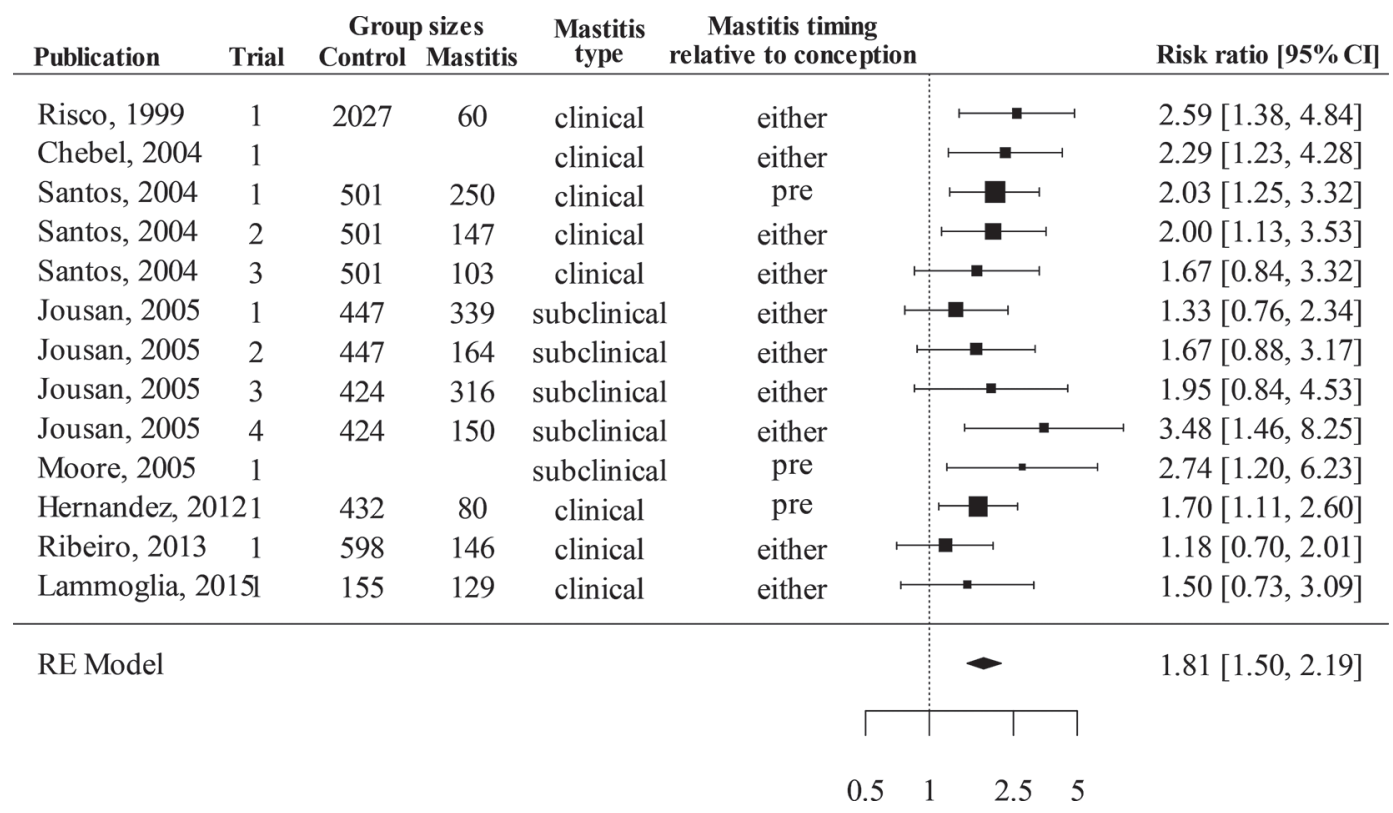

Figure 5. Forest plot to evaluate the effect of mastitis incidence on pregnancy loss in dairy cows. The effect size for each individual trial is reported and the size of the box for each trial indicates its weight in the pooled estimate. Mastitis incidence could have been clinical (identified by detection of abnormal milk, abnormal udder characteristics, or recorded treatment) or subclinical (identified by SCC evaluation or culture results). Estimates were obtained by random effects (RE) models (no moderators) for which the pooled estimate is presented as the solid polygon on the last row. Papers are listed by first author and year of publication. 
(a)

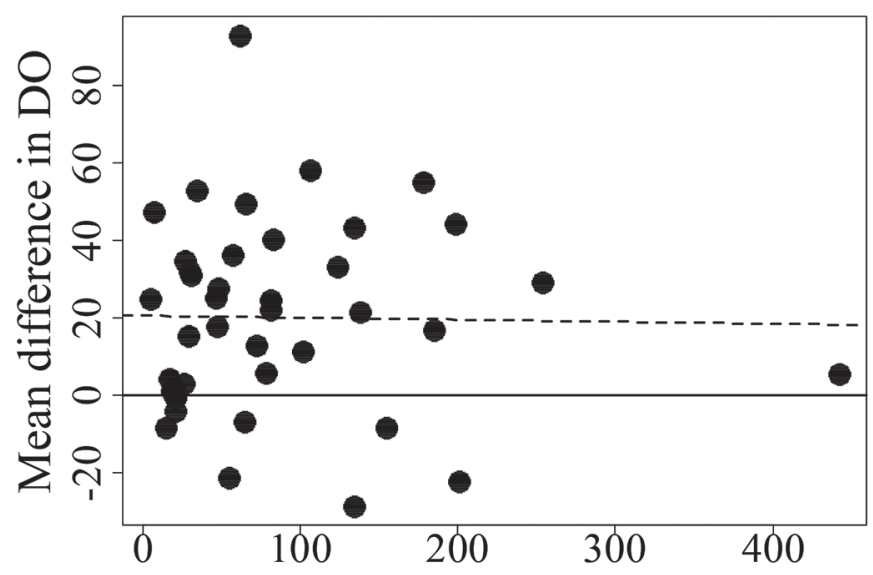

(c)

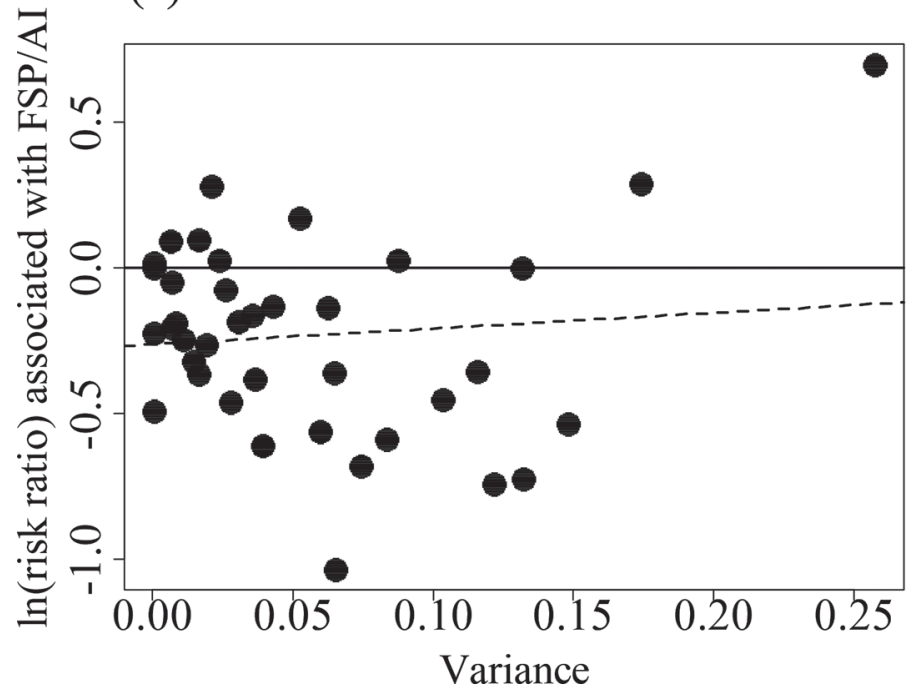

(b)

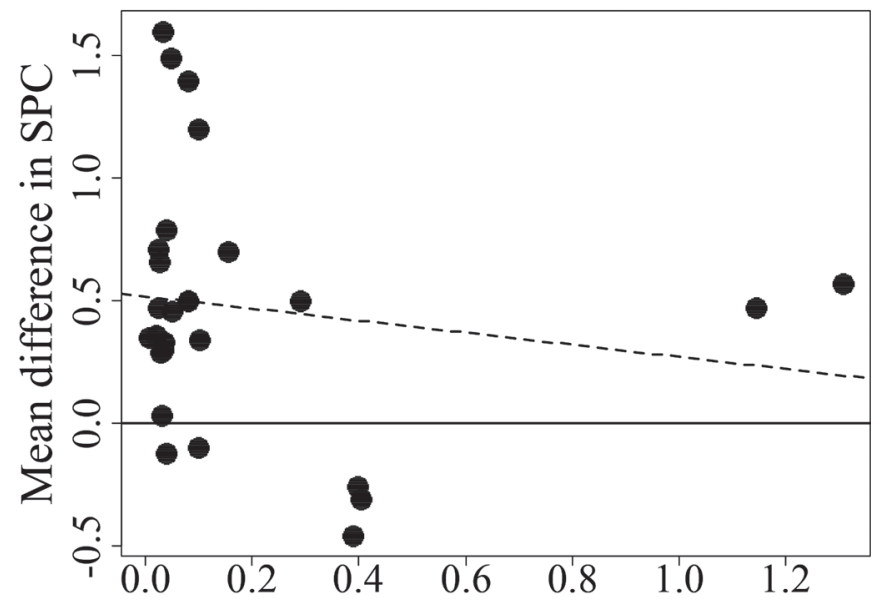

(d)

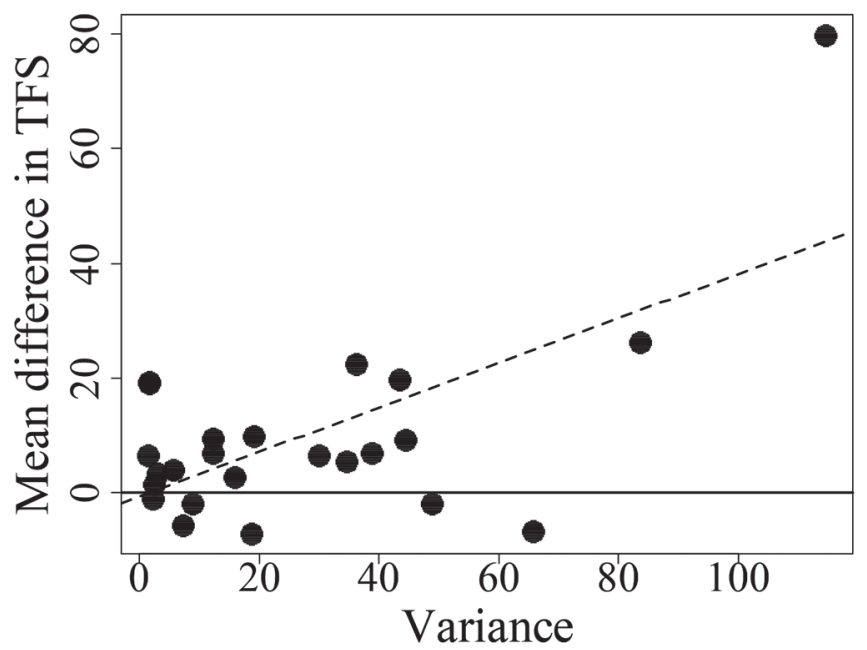

Figure 6. Egger's regression test for publication bias in a meta-analysis of trials assessing the effect of mastitis incidence on (a) days open (DO), (b) services per conception (SPC), (c) pregnancies per insemination at first service (FSP/AI), and (d) time to first service (TFS) in dairy cows. Individual points indicate individual trial effect size and the corresponding sampling variance. The dashed line shows the regression line of the effect sizes on the corresponding sampling variances.

post-first insemination, suggesting no difference on the risk of conceiving between cows with mastitis during this period and cows with no mastitis. However, when mastitis was diagnosed pre-first insemination or either pre- or post-first insemination, the risk ratio for FSP/AI suggested that cows with mastitis were at a lower risk of conceiving than cows with no mastitis, at the same period. It is important to note, however, that biologically, mastitis might have negative effects on pregnancy establishment both pre-insemination (e.g., by affecting the cow's physiological ability to become pregnant or oocyte development) and post-insemination (e.g., by interfering with embryo development). In this context, the time of diagnosis might not be the best indicator of the biological response of cows with mastitis on reproductive outcomes. This scenario becomes particularly problematic if mastitis is not overtly detected until post-first insemination, whereas the cow was already experiencing a physiological response to the infection before first insemination.

Mastitis diagnosis timing relative to the insemination leading to conception was also assessed for DO and SPC. In particular, for SPC, both mastitis diagnosis at pre- or either pre- or post-insemination leading to con- 
ception resulted in an increase in the mean difference between cows with mastitis and cows with no mastitis. These results are difficult to interpret because diagnosis timing categories were dictated by the variety of study designs used and, as a consequence, broadly defined. For example, very few trials considered mastitis diagnosis occurring only after the insemination leading to conception; therefore, those trials were grouped together with trials that considered mastitis diagnosis either before or after the insemination leading to conception. As a result, this category still included cows that were diagnosed with mastitis pre-insemination leading to conception, overlapping with the pre-insemination leading to conception category. Ideally, we would be able to differentiate mastitis diagnosis timing by days relative to specific events.

The effect of breed and study location were chosen as moderator variables because of their potential effect on both mastitis incidence and reproductive performance outcomes. Differences in susceptibility to mastitis, reproductive success, housing, nutrition, and other breed and management specific factors all have the ability to influence the relationship of interest in this study. Regardless, neither breed or study location were retained in the final models for any of the meta-regressions. This may have been observed because of the large amount of variation in management and study designs even within breed and study location categories.

Although some significant moderators were identified through meta-regression, high levels of heterogeneity relative to total variability remained even after metaregression analysis. One inherent problem with using observational studies in meta-analysis is that there are naturally high levels of heterogeneity between trials because of differences in study design and reporting (Shrier et al., 2007). Not only does this influence the level of heterogeneity within the meta-analysis, but it also limits the number of potential moderator variables that are available for exploration because not all studies report the same information. An updated meta-analysis that includes more detailed moderators would be beneficial as additional data become available. Other potential moderators that should be explored if reported in future studies include variation in the accuracy of mastitis identification, the bacterial cause of mastitis, if and how mastitis cases were treated, breeding method, breeding season, housing type, bedding type, and other cow factors that could potentially confound the strength of the relationship between mastitis incidence and reproductive performance (e.g., milk production level, parity group, BCS, incidence of other diseases). In addition, exploration of other sources of data could complement meta-analytic efforts by utilizing variables that are often not reported in peer-reviewed publication, for example, utilization of farm management records.

\section{Publication Bias and Influential Trials}

Both publication bias and influential trials could affect the reliability of meta-analysis findings. In this study, publication bias was identified in 4 of the metaanalysis models (TFS, DO, SPC, and FSP/AI). Although efforts were made to include all relevant studies, some studies were not included in the analysis because of their failure to report important data necessary for effect size calculations; this could have resulted in some inclusion bias. No influential trials were identified for any of the reproductive outcomes.

\section{CONCLUSIONS}

Based on the result from this meta-analysis, there is a quantifiable association between mastitis incidence and reproductive performance outcomes in dairy cattle. This finding emphasizes the importance of mastitis prevention to maximizing cow performance and farm profitability not only from a short-term milk production perspective but also from a reproductive efficiency standpoint. The construction of meta-regression models would allow quantification of how specific moderator variables effect the estimated effect sizes. However, given the low availability of studies that reported detailed herd characteristics, further efforts will be required to better quantitatively describe the mechanisms behind the effect of mastitis on reproductive outcomes in dairy herds.

\section{ACKNOWLEDGMENTS}

The authors appreciate the clarification of study methods, analysis, and results of the original studies used in this meta-analysis, as provided by Neal Schrick (University of Tennessee, Knoxville, TN), T. Nakao (Yamaguchi University, Yamaguchi, Japan), and Bouamra Mohamed (High National Veterinary School, Algiers, Algeria). This research was supported by state and federal funds appropriated to the Ohio Agricultural Research and Development Center, The Ohio State University (Columbus, OH).

\section{REFERENCES}

Ahmadzadeh, A., F. Frago, B. Shafii, J. Dalton, W. Price, and M. McGuire. 2009. Effect of clinical mastitis and other diseases on reproductive performance of Holstein cows. Anim. Reprod. Sci. 112:273-282. 
Barker, A., F. Schrick, M. Lewis, H. Dowlen, and S. Oliver. 1998. Influence of clinical mastitis during early lactation on reproductive performance of Jersey cows. J. Dairy Sci. 81:1285-1290.

Bouamra, M., F. Ghozlane, and M. Ghozlane. 2017. Factors affecting reproductive performance of dairy cow in Algeria: Effects of clinical mastitis. Afr. J. Biotechnol. 16:91-95.

Boujenane, I., J. El Aimani, and K. By. 2015. Effects of clinical mastitis on reproductive and milk performance of Holstein cows in Morocco. Trop. Anim. Health Prod. 47:207-211.

Chebel, R. C., J. E. Santos, J. P. Reynolds, R. L. Cerri, S. O. Juchem, and M. Overton. 2004. Factors affecting conception rate after artificial insemination and pregnancy loss in lactating dairy cows. Anim. Reprod. Sci. 84:239-255.

Chung, Y., S. Rabe-Hesketh, and I. H. Choi. 2013. Avoiding zero between-study variance estimates in random-effects meta-analysis. Stat. Med. 32:4071-4089.

Dahl, M. O., F. P. Maunsell, A. De Vries, K. N. Galvao, C. A. Risco, and J. A. Hernandez. 2017. Evidence that mastitis can cause pregnancy loss in dairy cows: A systematic review of observational studies. J. Dairy Sci. 100:8322-8329.

Egger, M., G. D. Smith, M. Schneider, and C. Minder. 1997. Bias in meta-analysis detected by a simple, graphical test. BMJ 315:629 634

Elmaghraby, M., T. El-Dien, K. Metwelly, and A. El-Sheikh. 2004. Influence of clinical mastitis during the pre-conception period on milk yield and reproductive performance of Holstein-Friesian cows. Minufiya Vet. J. 3:331-347.

Elmaghraby, M. M., A. F. El-Nahas, M. M. Fathala, F. M. Sahwan, and M. A. T. El-Dien. 2017. Incidence of clinical mastitis and its influence on reproductive performance of dairy cows. Alex. J. Vet. Sci. 54:84-91.

Fleischer, P., M. Metzner, M. Beyerbach, M. Hoedemaker, and W. Klee. 2001. The relationship between milk yield and the incidence of some diseases in dairy cows. J. Dairy Sci. 84:2025-2035.

Fourichon, C., H. Seegers, and X. Malher. 2000. Effect of disease on reproduction in the dairy cow: A meta-analysis. Theriogenology 53:1729-1759.

Fuenzalida, M., P. Fricke, and P. Ruegg. 2015. The association between occurrence and severity of subclinical and clinical mastitis on pregnancies per artificial insemination at first service of Holstein cows. J. Dairy Sci. 98:3791-3805.

Gunay, A., and U. Gunay. 2008. Effects of clinical mastitis on reproductive performance in Holstein cows. Acta Vet. Brno 77:555-560.

Hansen, P. J., P. Soto, and R. P. Natzke. 2004. Mastitis and fertility in cattle-Possible involvement of inflammation or immune activation in embryonic mortality. Am. J. Reprod. Immunol. 51:294-301.

Heringstad, B., X.-L. Wu, and D. Gianola. 2009. Inferring relationships between health and fertility in Norwegian Red cows using recursive models. J. Dairy Sci. 92:1778-1784.

Hernandez, J. A., C. A. Risco, F. S. Lima, and J. E. Santos. 2012 Observed and expected combined effects of clinical mastitis and low body condition on pregnancy loss in dairy cows. Theriogenology $77: 115-121$

Higgins, J. P., and S. G. Thompson. 2002. Quantifying heterogeneity in a meta-analysis. Stat. Med. 21:1539-1558.

Huszenicza, G., S. Jánosi, M. Kulcsar, P. Korodi, J. Reiczigel, L. Katai, A. Peters, and F. De Rensis. 2005. Effects of clinical mastitis on ovarian function in post-partum dairy cows. Reprod. Domest. Anim. 40:199-204.

Jousan, F. D., M. Drost, and P. Hansen. 2005. Factors associated with early and mid-to-late fetal loss in lactating and nonlactating Holstein cattle in a hot climate. J. Anim. Sci. 83:1017-1022.

Lammoglia, V., F. Hernández, R. Daniel, P. Zamora, M. Barrientos, and M. Domínguez. 2015. The effect of the most common diseases during the first 100 days of post partum on pregnancy rate and reproductive performance in the Mexican highlands dairy cows. Res. Opin. Anim. Vet. Sci. 5:370-374.

Lavon, Y., E. Ezra, G. Leitner, and D. Wolfenson. 2011. Association of conception rate with pattern and level of somatic cell count elevation relative to time of insemination in dairy cows. J. Dairy Sci. 94:4538-4545.
Lavon, Y., G. Leitner, H. Voet, and D. Wolfenson. 2010. Naturally occurring mastitis effects on timing of ovulation, steroid and gonadotrophic hormone concentrations, and follicular and luteal growth in cows. J. Dairy Sci. 93:911-921.

Lean, I. J., A. Rabiee, T. Duffield, and I. Dohoo. 2009. Invited review: Use of meta-analysis in animal health and reproduction: Methods and applications. J. Dairy Sci. 92:3545-3565.

Liberati, A., D. G. Altman, J. Tetzlaff, C. Mulrow, P. C. Gøtzsche, J. P. Ioannidis, M. Clarke, P. J. Devereaux, J. Kleijnen, and D. Moher. 2009. The PRISMA statement for reporting systematic reviews and meta-analyses of studies that evaluate health care interventions: Explanation and elaboration. PLoS Med. 6:e1000100.

Loeffler, S. H., M. De Vries, Y. Schukken, A. De Zeeuw, A. Dijkhuizen, F. De Graaf, and A. Brand. 1999. Use of AI technician scores for body condition, uterine tone and uterine discharge in a model with disease and milk production parameters to predict pregnancy risk at first AI in Holstein dairy cows. Theriogenology 51:1267-1284.

Lomander, H., C. Svensson, C. Hallén-Sandgren, H. Gustafsson, and J. Frössling. 2013. Associations between decreased fertility and management factors, claw health, and somatic cell count in Swedish dairy cows. J. Dairy Sci. 96:6315-6323.

Lucy, M. C. 2001. Reproductive loss in high-producing dairy cattle: Where will it end? J. Dairy Sci. 84:1277-1293.

McNally, J. C., M. A. Crowe, J. F. Roche, and M. E. Beltman. 2014. Effects of physiological and/or disease status on the response of postpartum dairy cows to synchronization of estrus using an intravaginal progesterone device. Theriogenology 82:1263-1272.

Moore, D. A., J. Cullor, R. Bondurant, and W. Sischo. 1991. Preliminary field evidence for the association of clinical mastitis with altered interestrus intervals in dairy cattle. Theriogenology $36: 257-265$.

Moher, D. A., A. Liberati, J. Tetzlaff, D. G. Altman, and P. Group. 2009. Preferred reporting items for systematic reviews and metaanalyses: The PRISMA statement. PLoS Med. 6:e1000097.

Moore, D. A., M. W. Overton, R. C. Chebel, M. L. Truscott, and R. H. BonDurant. 2005. Evaluation of factors that affect embryonic loss in dairy cattle. J. Am. Vet. Med. Assoc. 226:1112-1118.

Nguyen, T. C., T. Nakao, G. Gautam, L. Su, R. Ranasinghe, and M. Yusuf. 2011. Relationship between milk somatic cell count and postpartum ovarian cyclicity and fertility in dairy cows. Acta Vet. Hung. 59:349-362.

Perrin, L., R. Bostelmann, and I. Sheldon. 2007. Reduced conception rates associated with bovine mastitis during a 'window of opportunity'. Vet. Rec. 161:61-62.

Petersson, K.-J., E. Strandberg, H. Gustafsson, and B. Berglund. 2006. Environmental effects on progesterone profile measures of dairy cow fertility. Anim. Reprod. Sci. 91:201-214.

Rahman, M. M., M. Mazzilli, G. Pennarossa, T. Brevini, A. Zecconi, and F. Gandolfi. 2012. Chronic mastitis is associated with altered ovarian follicle development in dairy cattle. J. Dairy Sci. 95:18851893.

Ribeiro, E. S., F. Lima, L. Greco, R. Bisinotto, A. Monteiro, M. Favoreto, H. Ayres, R. Marsola, N. Martinez, and W. Thatcher. 2013. Prevalence of periparturient diseases and effects on fertility of seasonally calving grazing dairy cows supplemented with concentrates. J. Dairy Sci. 96:5682-5697.

Risco, C. A., G. Donovan, and J. Hernandez. 1999. Clinical mastitis associated with abortion in dairy cows. J. Dairy Sci. 82:1684-1689.

Santos, J. E., R. Cerri, M. Ballou, G. Higginbotham, and J. Kirk. 2004. Effect of timing of first clinical mastitis occurrence on lactational and reproductive performance of Holstein dairy cows. Anim. Reprod. Sci. 80:31-45.

Schams, D., and B. Berisha. 2004. Regulation of corpus luteum function in cattle-An overview. Reprod. Domest. Anim. 39:241-251.

Schrick, F. N., M. Hockett, A. Saxton, M. Lewis, H. Dowlen, and S. Oliver. 2001. Influence of subclinical mastitis during early lactation on reproductive parameters. J. Dairy Sci. 84:1407-1412.

Shrier, I., J.-F. Boivin, R. J. Steele, R. W. Platt, A. Furlan, R. Kakuma, J. Brophy, and M. Rossignol. 2007. Should meta-analyses of interventions include observational studies in addition to ran- 
domized controlled trials? A critical examination of underlying principles. Am. J. Epidemiol. 166:1203-1209.

Smulski, S., E. Malinowski, H. Markiewicz, and M. Kaczmarowski. 2006. The effect of mastitis on reproduction performance. Slovenian Veterinary Research 43:80-82.

Soto, P., R. Natzke, and P. Hansen. 2003. Identification of possible mediators of embryonic mortality caused by mastitis: Actions of lipopolysaccharide, prostaglandin $\mathrm{F} 2 \alpha$, and the nitric oxide generator, sodium nitroprusside dihydrate, on oocyte maturation and embryonic development in cattle. Am. J. Reprod. Immunol. 50:263-272.
Tillard, E., P. Humblot, B. Faye, P. Lecomte, I. Dohoo, and F. Bocquier. 2008. Postcalving factors affecting conception risk in Holstein dairy cows in tropical and sub-tropical conditions. Theriogenology 69:443-457.

Viechtbauer, W. 2010. Conducting meta-analyses in R with the metafor package. J. Stat. Softw. 36:1-48.

Yang, F. L., X. S. Li, B. Z. Yang, Y. Zhang, X. F. Zhang, G. S. Qin, and X. W. Liang. 2012. Clinical mastitis from calving to next conception negatively affected reproductive performance of dairy cows in Nanning, China. Afr. J. Biotechnol. 11:2574-2580.

\section{APPENDIX}

Table A1. Summary of trial sizes and mean \pm SD outcomes from studies used in a meta-analysis of the effects of mastitis incidence on time to first service (TFS)

\begin{tabular}{|c|c|c|c|c|c|c|}
\hline \multirow[b]{2}{*}{ Publication } & \multirow[b]{2}{*}{$\begin{array}{l}\text { Trial } \\
\text { no. }\end{array}$} & \multicolumn{2}{|r|}{ Control cows } & \multicolumn{2}{|r|}{ Mastitis cows } & \multirow[b]{2}{*}{$\begin{array}{l}\text { Uncertainty } \\
\text { type }\end{array}$} \\
\hline & & $\mathrm{n}$ & $\begin{array}{c}\text { TFS } \\
\left(\text { mean } \pm \text { uncertainty }^{1}\right)\end{array}$ & $\mathrm{n}$ & $\begin{array}{c}\text { TFS } \\
\left(\text { mean } \pm \text { uncertainty }^{1}\right)\end{array}$ & \\
\hline \multirow[t]{3}{*}{ Schrick et al. (2001) } & 1 & 326 & $67.8 \pm 2.2$ & 145 & $77.3 \pm 2.7$ & SEM \\
\hline & 2 & 326 & $67.8 \pm 2.2$ & 41 & $70.6 \pm 3.3$ & SEM \\
\hline & 3 & 326 & $67.8 \pm 2.2$ & 229 & $74.8 \pm 2.7$ & SEM \\
\hline \multirow[t]{3}{*}{ Santos et al. (2004) } & 1 & 501 & $64.0 \pm 1.4$ & 250 & $68.0 \pm 1.9$ & SEM \\
\hline & 2 & 501 & $64.0 \pm 1.4$ & 147 & $58.5 \pm 2.3$ & SEM \\
\hline & 3 & 501 & $64.0 \pm 1.4$ & 103 & $62.3 \pm 2.6$ & SEM \\
\hline \multirow[t]{2}{*}{ Smulski et al. (2006) } & 1 & 31 & $83.7 \pm 20.9$ & 35 & $90.8 \pm 29.4$ & SD \\
\hline & 2 & 31 & $83.7 \pm 20.9$ & 24 & $103.6 \pm 26.5$ & SD \\
\hline Perrin et al. (2007) & 1 & 24 & $83.2 \pm 6.1$ & 24 & $81.4 \pm 3.4$ & SE \\
\hline Gunay and Gunay (2008) & 1 & 45 & $75.9 \pm 6.3$ & 45 & $95.2 \pm 5.4$ & SD \\
\hline \multirow{2}{*}{ Boujenane et al. (2015) } & 2 & 1,261 & $92.5 \pm 1.8$ & 73 & $101.8 \pm 6.4$ & $\mathrm{SE}$ \\
\hline & 3 & 1,261 & $92.5 \pm 1.8$ & 130 & $98.0 \pm 5.6$ & $\mathrm{SE}$ \\
\hline Lammoglia et al. (2015) & 1 & 155 & $77.6 \pm 2.9$ & 129 & $70.5 \pm 3.2$ & SEM \\
\hline \multirow{2}{*}{ Bouamra et al. (2017) } & 1 & 316 & $111.0 \pm 5.4$ & 62 & $137.3 \pm 7.4$ & SEM \\
\hline & 2 & 316 & $111.0 \pm 5.4$ & 54 & $190.9 \pm 9.2$ & SEM \\
\hline Elmaghraby et al. (2017) & 1 & 1,228 & $66.9 \pm 0.4$ & 647 & $73.4 \pm 1.1$ & $\mathrm{SE}$ \\
\hline
\end{tabular}

\footnotetext{
${ }^{1}$ As reported in the original publication.
} 
Table A2. Summary of trial sizes and mean outcomes from studies used in a meta-analysis of the effects of mastitis incidence on days open (DO)

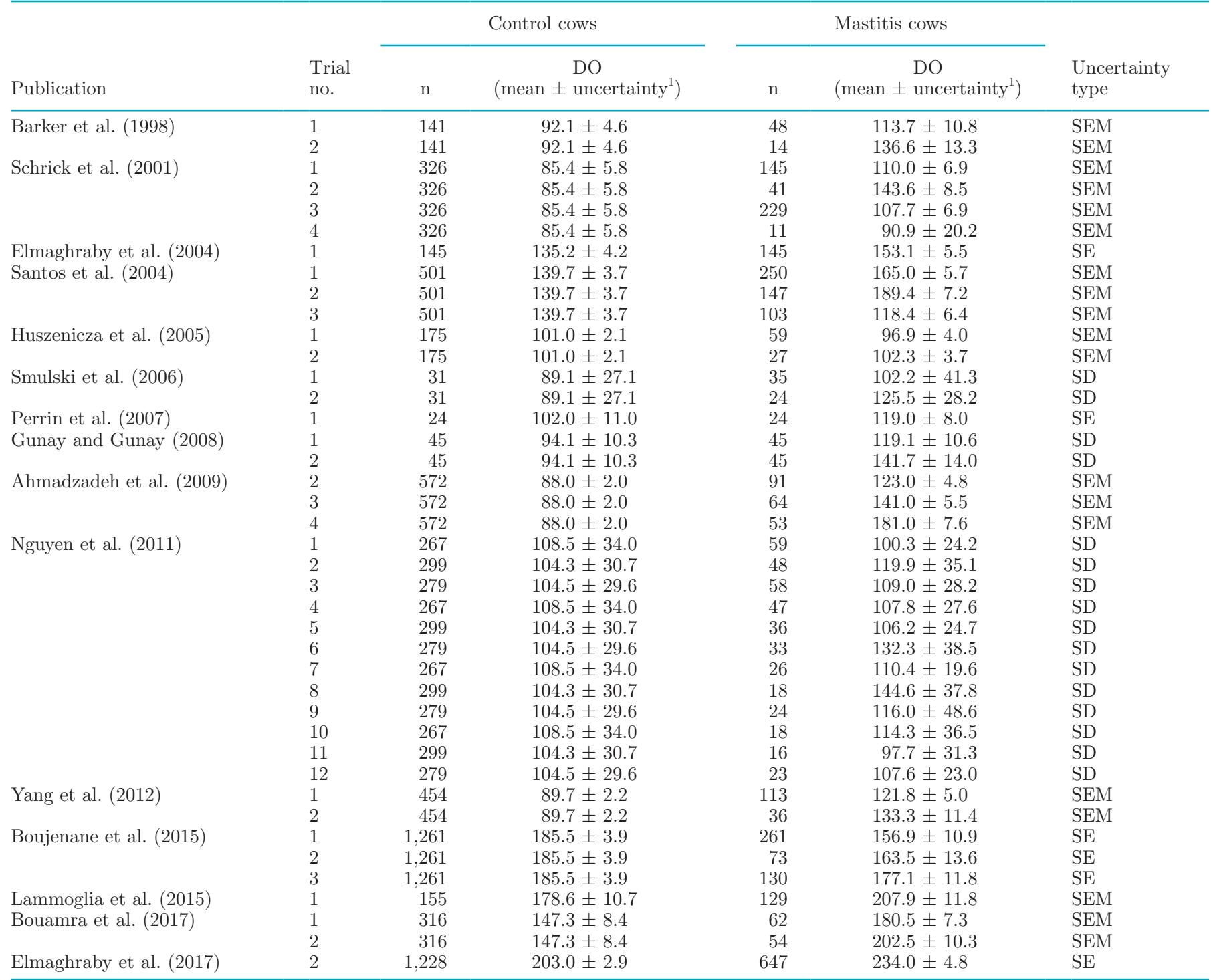

${ }^{1}$ As reported in the original publication. 
Table A3. Summary of trial sizes and mean outcomes from studies used in a meta-analysis of the effects of mastitis incidence on services per conception (SPC)

\begin{tabular}{|c|c|c|c|c|c|c|}
\hline \multirow[b]{2}{*}{ Publication } & \multirow[b]{2}{*}{$\begin{array}{l}\text { Trial } \\
\text { no. }\end{array}$} & \multicolumn{2}{|r|}{ Control cows } & \multicolumn{2}{|r|}{ Mastitis cows } & \multirow[b]{2}{*}{$\begin{array}{l}\text { Uncertainty } \\
\text { type }\end{array}$} \\
\hline & & $\mathrm{n}$ & 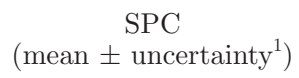 & $\mathrm{n}$ & 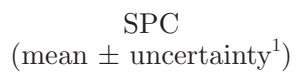 & \\
\hline \multirow[t]{2}{*}{ Barker et al. (1998) } & 1 & 143 & $1.70 \pm 0.10$ & 46 & $1.60 \pm 0.30$ & SEM \\
\hline & 2 & 143 & $1.70 \pm 0.10$ & 13 & $2.90 \pm 0.30$ & SEM \\
\hline \multirow[t]{4}{*}{ Schrick et al. (2001) } & 1 & 326 & $1.60 \pm 0.20$ & 145 & $2.10 \pm 0.20$ & SEM \\
\hline & 2 & 326 & $1.60 \pm 0.20$ & 41 & $3.00 \pm 0.20$ & SEM \\
\hline & 3 & 326 & $1.60 \pm 0.20$ & 229 & $2.10 \pm 0.20$ & SEM \\
\hline & 4 & 326 & $1.60 \pm 0.20$ & 11 & $2.10 \pm 0.50$ & SEM \\
\hline Elmaghraby et al. (2004) & 1 & 145 & $2.59 \pm 0.10$ & 145 & $2.92 \pm 0.16$ & $\mathrm{SE}$ \\
\hline \multirow[t]{3}{*}{ Santos et al. (2004) } & 1 & 501 & $2.59 \pm 0.10$ & 250 & $2.62 \pm 0.14$ & SEM \\
\hline & 2 & 501 & $2.59 \pm 0.10$ & 147 & $3.05 \pm 0.20$ & SEM \\
\hline & 3 & 501 & $2.59 \pm 0.10$ & 103 & $2.47 \pm 0.17$ & SEM \\
\hline \multirow[t]{2}{*}{ Smulski et al. (2006) } & 1 & 31 & $1.25 \pm 0.51$ & 35 & $1.54 \pm 0.85$ & $\mathrm{SD}$ \\
\hline & 2 & 31 & $1.25 \pm 0.51$ & 24 & $2.04 \pm 0.85$ & $\mathrm{SD}$ \\
\hline Perrin et al. (2007) & 1 & 24 & $1.70 \pm 0.23$ & 24 & $2.04 \pm 0.22$ & $\mathrm{SE}$ \\
\hline \multirow[t]{2}{*}{ Gunay and Gunay (2008) } & 1 & 45 & $1.80 \pm 0.80$ & 45 & $2.10 \pm 0.90$ & $\mathrm{SD}$ \\
\hline & 2 & 45 & $1.80 \pm 0.80$ & 45 & $3.40 \pm 0.90$ & $\mathrm{SD}$ \\
\hline \multirow[t]{3}{*}{ Ahmadzadeh et al. (2009) } & 2 & 572 & $1.62 \pm 0.05$ & 91 & $1.98 \pm 0.13$ & SEM \\
\hline & 3 & 572 & $1.62 \pm 0.05$ & 64 & $2.33 \pm 0.15$ & SEM \\
\hline & 4 & 572 & $1.62 \pm 0.05$ & 53 & $3.11 \pm 0.21$ & SEM \\
\hline \multirow[t]{2}{*}{ Yang et al. (2012) } & 1 & 454 & $1.53 \pm 0.03$ & 113 & $1.88 \pm 0.08$ & SEM \\
\hline & 2 & 454 & $1.53 \pm 0.03$ & 36 & $2.19 \pm 0.16$ & SEM \\
\hline \multirow[t]{3}{*}{ Boujenane et al. (2015) } & 1 & 1,261 & $2.48 \pm 0.60$ & 261 & $2.02 \pm 0.17$ & $\mathrm{SE}$ \\
\hline & 2 & 1,261 & $2.48 \pm 0.60$ & 73 & $2.17 \pm 0.21$ & $\mathrm{SE}$ \\
\hline & 3 & 1,261 & $2.48 \pm 0.60$ & 130 & $2.22 \pm 0.19$ & $\mathrm{SE}$ \\
\hline Lammoglia et al. (2015) & 1 & 155 & $3.00 \pm 0.18$ & 129 & $3.70 \pm 0.35$ & SEM \\
\hline \multirow{2}{*}{ Bouamra et al. (2017) } & 1 & 316 & $1.47 \pm 0.65$ & 62 & $1.94 \pm 0.85$ & SEM \\
\hline & 2 & 316 & $1.47 \pm 0.65$ & 54 & $2.04 \pm 0.94$ & SEM \\
\hline Elmaghraby et al. (2017) & 2 & 1,228 & $4.65 \pm 0.08$ & 647 & $5.12 \pm 0.13$ & $\mathrm{SE}$ \\
\hline
\end{tabular}

${ }^{1}$ As reported in the original publication. 
Table A4. Summary of trial sizes and mean outcomes from studies used in a meta-analysis of the effects of mastitis incidence on pregnancies per insemination at first service (FSP/AI)

\begin{tabular}{|c|c|c|c|c|c|}
\hline \multirow[b]{2}{*}{ Publication } & \multirow[b]{2}{*}{$\begin{array}{l}\text { Trial } \\
\text { no. }\end{array}$} & \multicolumn{2}{|c|}{ Control cows } & \multicolumn{2}{|c|}{ Mastitis cows } \\
\hline & & $\mathrm{n}$ & $\begin{array}{c}\mathrm{FSP} / \mathrm{AI} \\
(\text { mean })\end{array}$ & $\mathrm{n}$ & $\begin{array}{c}\mathrm{FSP} / \mathrm{AI} \\
\text { (mean) }\end{array}$ \\
\hline Loeffler et al. (1999) & 1 & 1,404 & 46.2 & 146 & 33.6 \\
\hline \multirow[t]{3}{*}{ Santos et al. (2004) } & 1 & 501 & 28.7 & 250 & 22.1 \\
\hline & 2 & 501 & 28.7 & 147 & 10.2 \\
\hline & 3 & 501 & 28.7 & 103 & 37.9 \\
\hline Petersson et al. (2006) & 1 & 642 & 39.5 & 94 & 43.4 \\
\hline \multirow[t]{4}{*}{ Perrin et al. (2007) } & 1 & 24 & 63.0 & 24 & 30.0 \\
\hline & 2 & 17 & 35.3 & 17 & 47.1 \\
\hline & 3 & 22 & 40.9 & 22 & 40.9 \\
\hline & 4 & 17 & 23.5 & 17 & 47.1 \\
\hline \multirow{2}{*}{ Tillard et al. (2008) } & 1 & 413 & 26.6 & 33 & 27.3 \\
\hline & 2 & 729 & 26.9 & 134 & 27.6 \\
\hline Heringstad et al. (2009) & 1 & 50,011 & 67.0 & 5,557 & 68.0 \\
\hline Lavon et al. (2011) & 1 & 178,512 & 39.4 & 46,374 & 31.5 \\
\hline \multirow[t]{12}{*}{ Nguyen et al. (2011) } & 1 & 267 & 47.6 & 59 & 44.1 \\
\hline & 2 & 299 & 47.5 & 48 & 27.1 \\
\hline & 3 & 279 & 47.7 & 58 & 39.7 \\
\hline & 4 & 267 & 47.6 & 47 & 40.4 \\
\hline & 5 & 299 & 47.5 & 36 & 41.7 \\
\hline & 6 & 279 & 47.7 & 33 & 33.3 \\
\hline & 7 & 267 & 47.6 & 26 & 23.1 \\
\hline & 8 & 299 & 47.5 & 18 & 27.8 \\
\hline & 9 & 279 & 47.7 & 24 & 41.7 \\
\hline & 10 & 267 & 47.6 & 18 & 33.3 \\
\hline & 11 & 299 & 47.5 & 16 & 56.3 \\
\hline & 12 & 279 & 47.7 & 23 & 30.4 \\
\hline \multirow{2}{*}{ Yang et al. (2012) } & 1 & 454 & 54.9 & 113 & 38.1 \\
\hline & 2 & 454 & 54.9 & 36 & 27.8 \\
\hline \multirow{2}{*}{ Lomander et al. (2013) } & 1 & 13,619 & 49.0 & 45,356 & 49.0 \\
\hline & 2 & 43,943 & 54.0 & 15,032 & 33.0 \\
\hline Ribeiro et al. (2013) & 1 & 598 & 66.9 & 146 & 54.5 \\
\hline McNally et al. (2014) & 1 & 154 & 53.9 & 30 & 30.0 \\
\hline \multirow[t]{5}{*}{ Fuenzalida et al. (2015) } & 1 & 2,103 & 41.0 & 221 & 45.0 \\
\hline & 2 & 2,103 & 41.0 & 207 & 32.0 \\
\hline & 3 & 2,103 & 41.0 & 72 & 28.0 \\
\hline & 4 & 2,103 & 41.0 & 271 & 34.0 \\
\hline & 5 & 2,103 & 41.0 & 270 & 39.0 \\
\hline \multirow[t]{2}{*}{ Bouamra et al. (2017) } & 1 & 316 & 61.4 & 62 & 38.7 \\
\hline & 2 & 316 & 61.4 & 54 & 33.3 \\
\hline
\end{tabular}

Table A5. Summary of trial sizes and mean outcomes from studies used in a meta-analysis of the effects of mastitis incidence on pregnancy loss (PL)

\begin{tabular}{|c|c|c|c|c|c|}
\hline \multirow[b]{2}{*}{ Publication } & \multirow[b]{2}{*}{$\begin{array}{l}\text { Trial } \\
\text { no. }\end{array}$} & \multicolumn{2}{|c|}{ Control cows } & \multicolumn{2}{|c|}{ Mastitis cows } \\
\hline & & $\mathrm{n}$ & $\begin{array}{c}\mathrm{PL} \\
(\text { mean })\end{array}$ & $\mathrm{n}$ & $\begin{array}{c}\mathrm{PL} \\
\text { (mean) }\end{array}$ \\
\hline Risco et al. (1999) & 1 & 2,027 & 5.8 & 60 & 15.0 \\
\hline Chebel et al. (2004) & 1 & $\mathrm{NA}^{1}$ & 12.3 & NA & 22.6 \\
\hline \multirow[t]{3}{*}{ Santos et al. (2004) } & 1 & 501 & 5.8 & 250 & 11.8 \\
\hline & 2 & 501 & 5.8 & 147 & 11.6 \\
\hline & 3 & 501 & 5.8 & 103 & 9.7 \\
\hline \multirow[t]{4}{*}{ Jousan et al. (2005) } & 1 & 447 & 5.1 & 339 & 6.8 \\
\hline & 2 & 447 & 5.1 & 164 & 8.5 \\
\hline & 3 & 424 & 2.1 & 316 & 4.1 \\
\hline & 4 & 424 & 2.1 & 150 & 7.3 \\
\hline Moore et al. (2005) & 1 & NA & 12.8 & NA & 31.0 \\
\hline Hernandez et al. (2012) & 1 & 432 & 15.5 & 80 & 26.3 \\
\hline Ribeiro et al. (2013) & 1 & 598 & 9.2 & 146 & 10.9 \\
\hline Lammoglia et al. (2015) & 1 & 155 & 7.7 & 129 & 11.6 \\
\hline
\end{tabular}

${ }^{1} \mathrm{NA}=$ not available. 


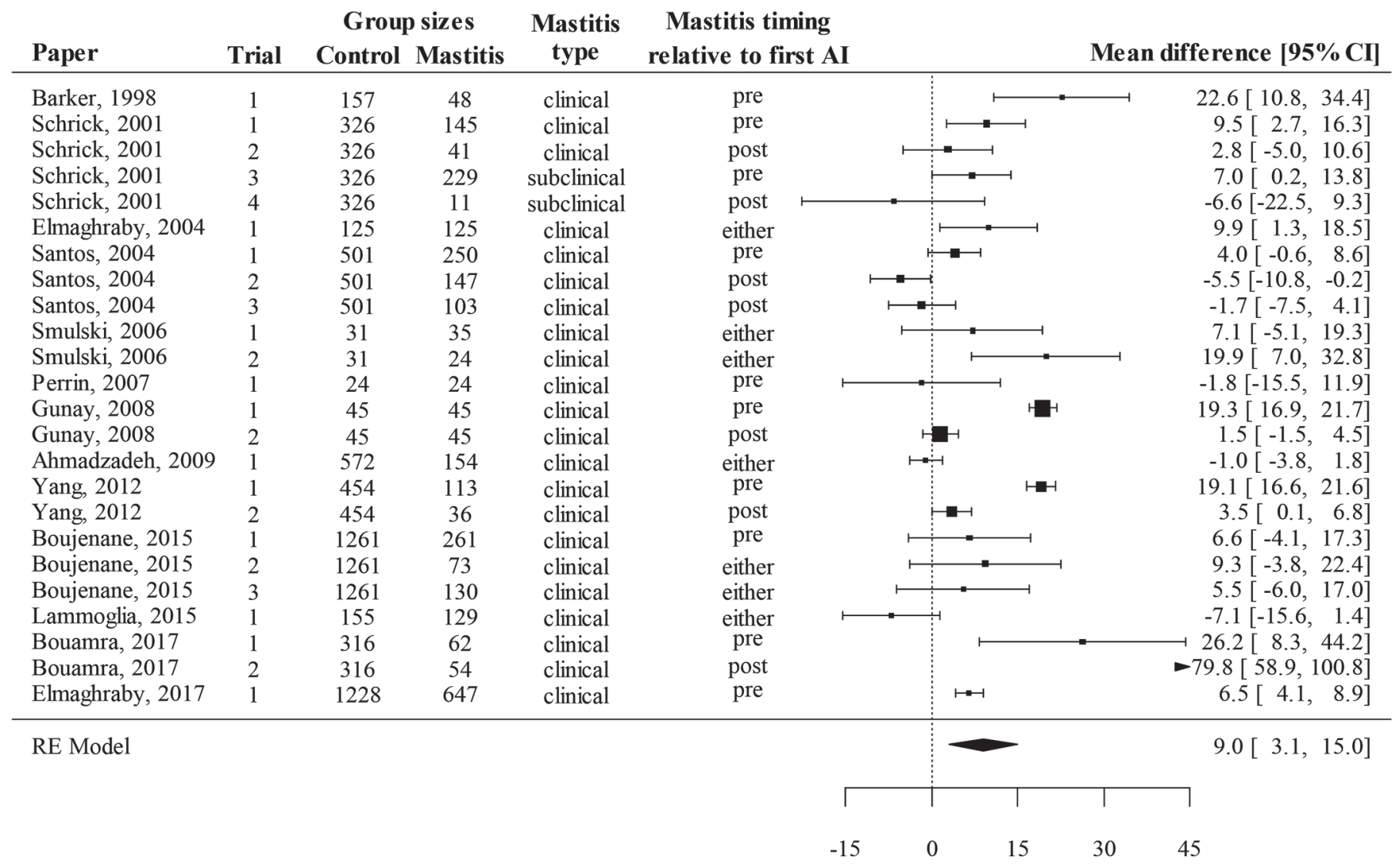

Figure A1. Forest plot to evaluate the effect of mastitis incidence on time to first service in dairy cows. The effect size for each individual trial is reported and the size of the box for each trial indicates its weight in the pooled estimate. Mastitis incidence could have been clinical (identified by detection of abnormal milk, abnormal udder characteristics, or recorded treatment) or subclinical (identified by SCC evaluation or culture results). Estimates were obtained by random effects (RE) models (no moderators) for which the pooled estimate is a solid polygon presented in the last row. Papers are listed by first author and year of publication. 


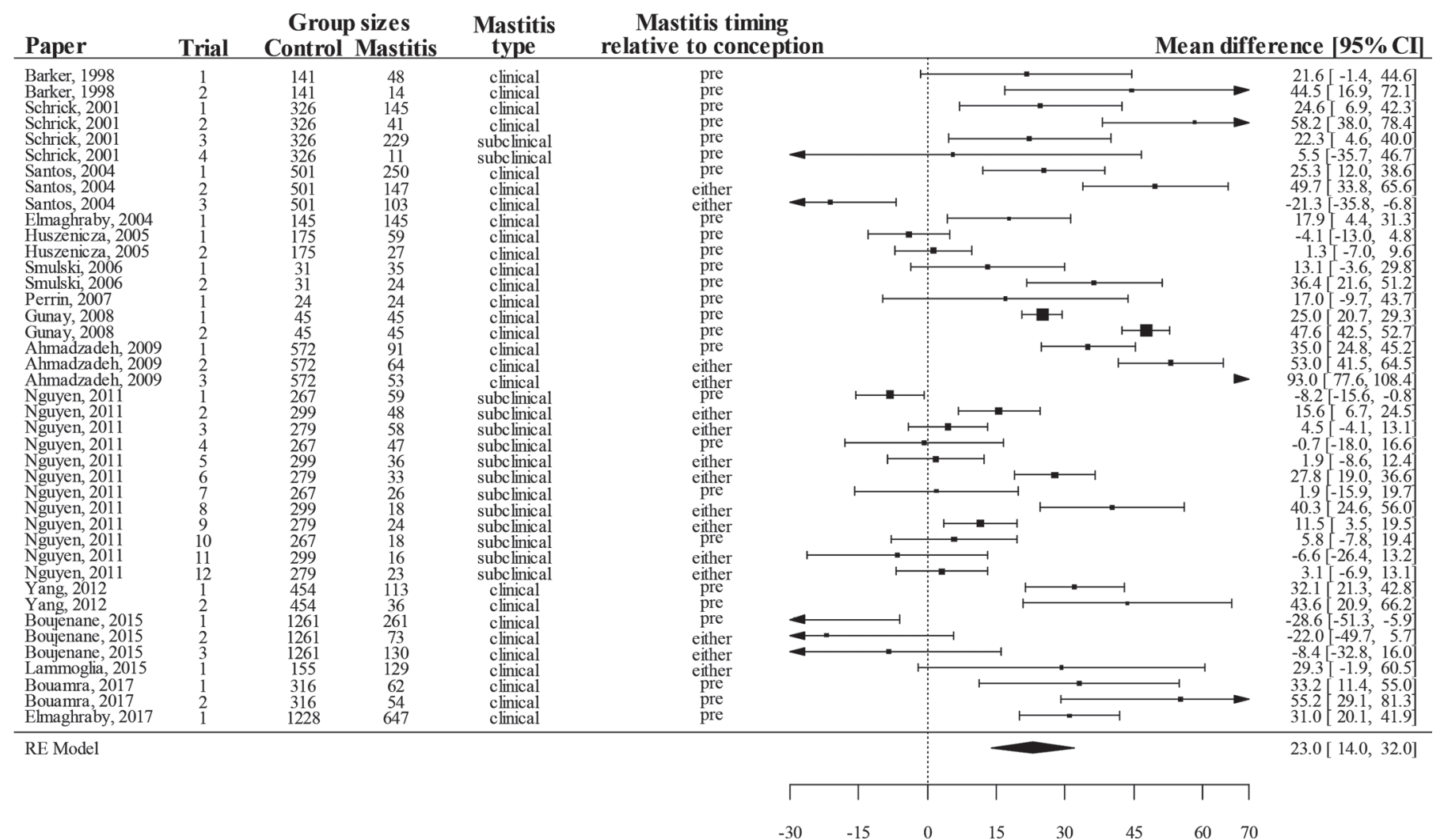

Figure A2. Forest plot to evaluate the effect of mastitis incidence on days open in dairy cows. The effect size for each individual trial is reported and the size of the box for each trial indicates its weight in the pooled estimate. Mastitis incidence could have been clinical (identified by detection of abnormal milk, abnormal udder characteristics, or recorded treatment) or subclinical (identified by SCC evaluation or culture results). Estimates were obtained by random effects (RE) models (no moderators) for which the pooled estimate is a solid polygon presented in the last row. Papers are listed by first author and year of publication. 


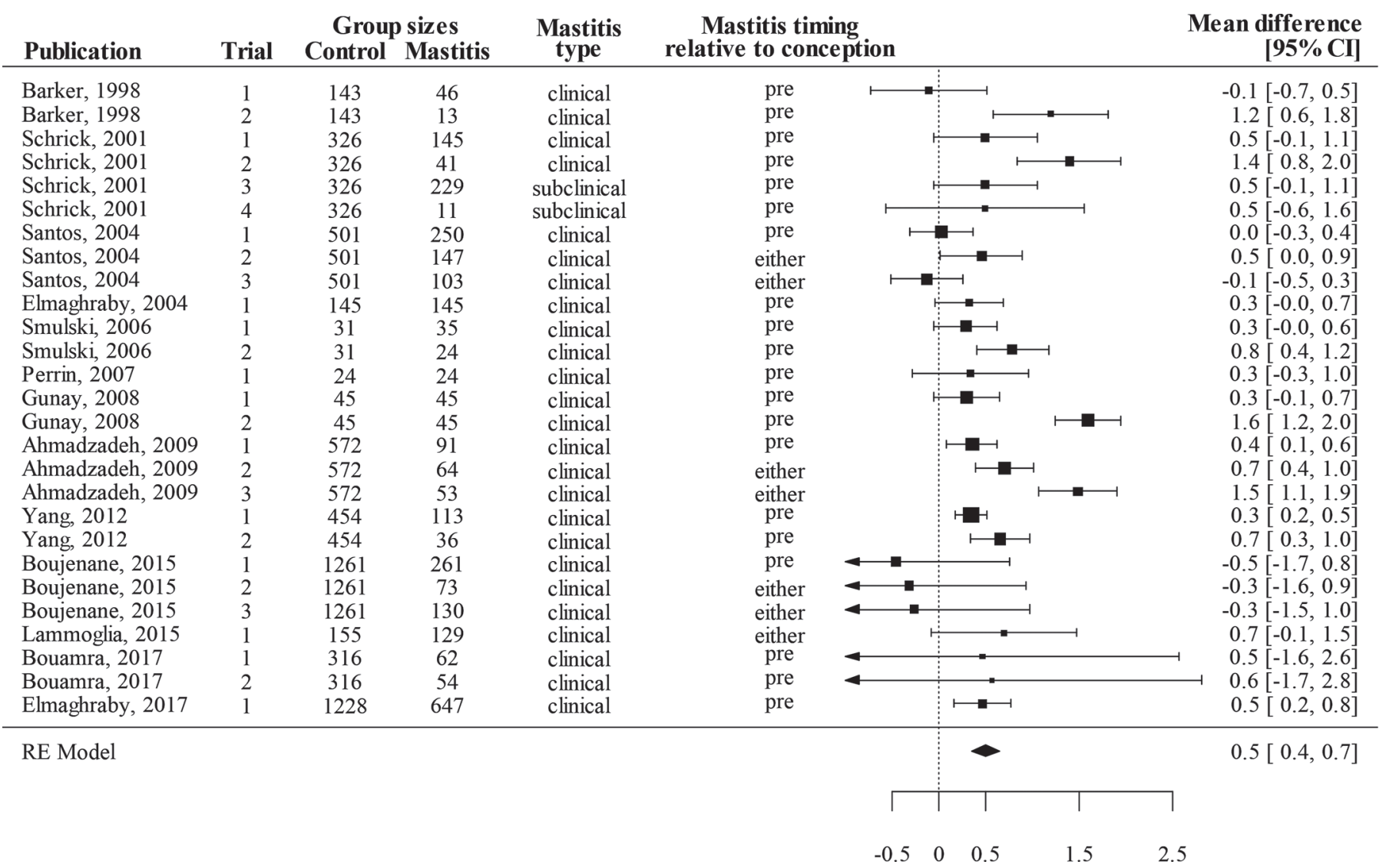

Figure A3. Forest plot to evaluate the effect of mastitis incidence on services per conception in dairy cows. The effect size for each individual trial is reported and the size of the box for each trial indicates its weight in the pooled estimate. Mastitis incidence could have been clinical (identified by detection of abnormal milk, abnormal udder characteristics, or recorded treatment) or subclinical (identified by SCC evaluation or culture results). Estimates were obtained by random effects (RE) models (no moderators) for which the pooled estimate is a solid polygon presented in the last row. Papers are listed by first author and year of publication. 


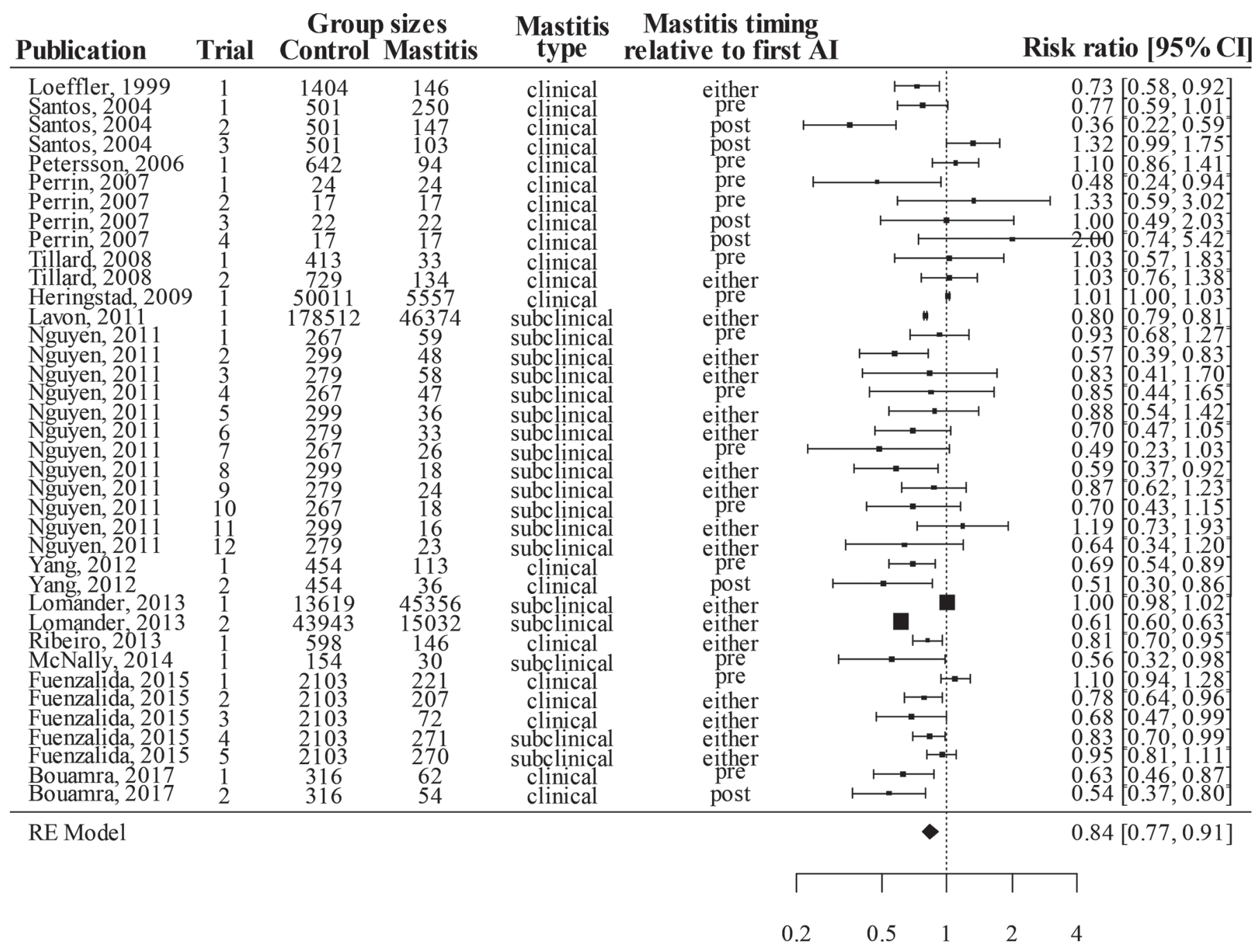

Figure A4. Forest plot to evaluate the effect of mastitis incidence on pregnancies per insemination at first service in dairy cows estimated using random effects models. The effect size for each individual trial is reported and the size of the box for each trial indicates its weight in the pooled estimate. Mastitis incidence could have been clinical (identified by detection of abnormal milk, abnormal udder characteristics, or recorded treatment) or subclinical (identified by SCC evaluation or culture results). Estimate was obtained by random effects (RE) models (no moderators) for which the pooled estimate is a solid polygon presented in the last row. Papers are listed by first author and year of publication. 